TID-8200(15th Rev.)

REACTOR TECHNOLOGY

I

MASTE:

\title{
NUCLEAR REACTORS
} BUILT,

BEING BUILT,

or PLANNED

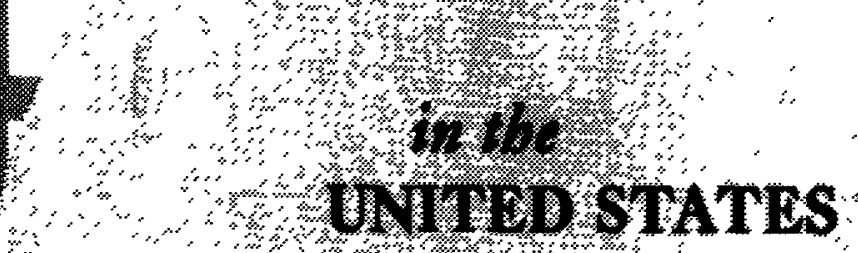

\%

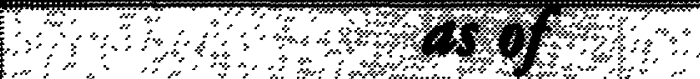

樂,

. Thepared by oflce of the

a $\quad 2 \%$

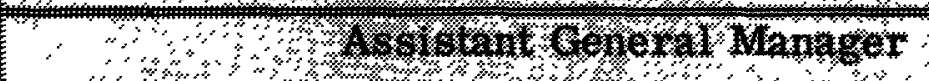

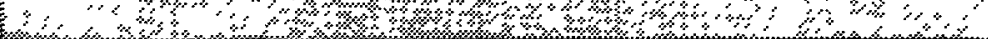

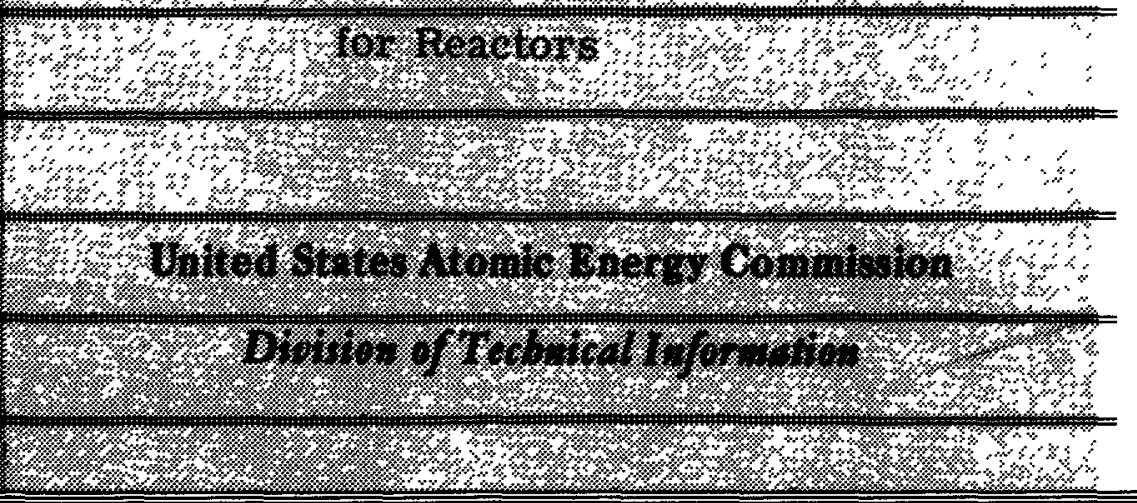

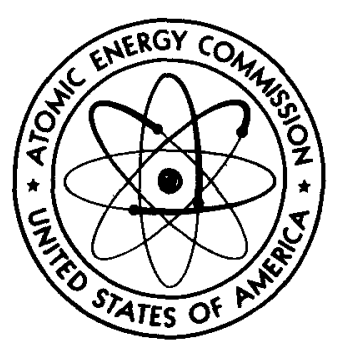




\section{DISCLAIMER}

This report was prepared as an account of work sponsored by an agency of the United States Government. Neither the United States Government nor any agency Thereof, nor any of their employees, makes any warranty, express or implied, or assumes any legal liability or responsibility for the accuracy, completeness, or usefulness of any information, apparatus, product, or process disclosed, or represents that its use would not infringe privately owned rights. Reference herein to any specific commercial product, process, or service by trade name, trademark, manufacturer, or otherwise does not necessarily constitute or imply its endorsement, recommendation, or favoring by the United States Government or any agency thereof. The views and opinions of authors expressed herein do not necessarily state or reflect those of the United States Government or any agency thereof. 


\section{DISCLAIMER}

Portions of this document may be illegible in electronic image products. Images are produced from the best available original document. 
Price $\$ 3.00$. Available from the Clearinghouse for Federal Scientific and Technical Information, National Bureau of Standards, U. S. Department of Commerce, Springfield, Virginia 22151 


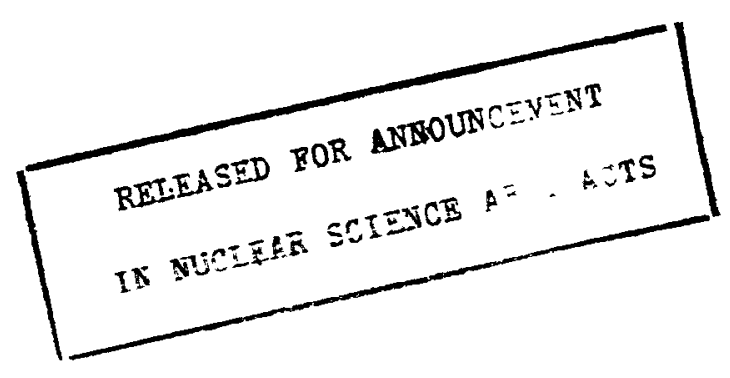

NUCLEAR REACTORS

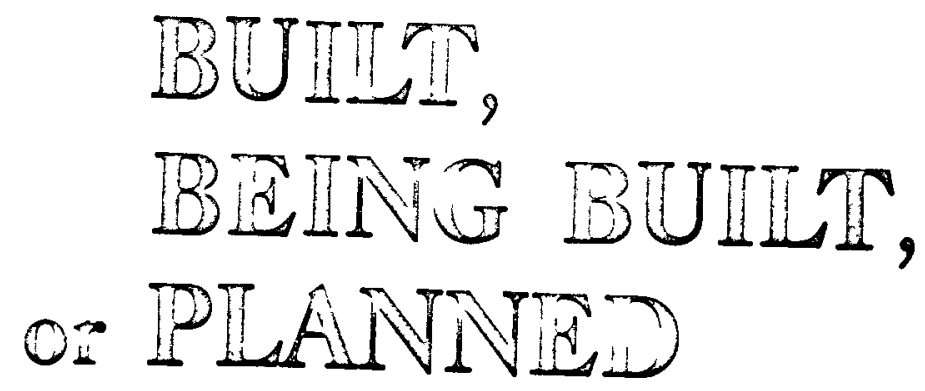

$\operatorname{tin} 1967$

LEG A L L NO report was prepared as an account of Government I CE

racy, completeness, or unt, or representation acting on behalf of the work Neither the United

of any information, ar usefulness of the infor, expressed or of the Commissither the United

privately owned an, apparatus, of the information or implied, with resion

B Assumes ans, or

use of any informatillabilities with respect

As used in the abparatus, methoct to the use of, or for theport may not infringe

ployee or contract above. "person act, or process of, or for damages resulting

such employee or contractormission, or behalf of the Com in this report

with the Commi or provides access to Commission, or ef such contractor. includes any em-

the commission, or his emps to, any information puployee of such cor, to the extent em -

with such contractor to his employment or prepares, 
NUCLEAR REACTORS

\author{
BUILT, \\ BEING BUILT, \\ or PLANNED
}




\section{FOREWORD}

This complation contains unclassified information about facilities built, being built, or planned in the United States as of Dec. 31,1966 , which are capable of sustaining a nuclear chain reaction. Certain projects relating to military and space systems are not listed in detall because of their classified nature.

Information is presented in five parts, each of which is categorized by primary function or purpose. The major parts, namely, civilian, military, production, and export, as well as such categories as power and propulsion, are self-explanatory. The major parts of the tabulation are indicated in the statistical summary on page 3. Various classes of reactors within these categories are defined as follows:

COMMERCIAL NUCLEAR POWER PLANT A nuclear facility constructed and operated under a license issued under Section 103 of the Atomic Energy Act of 1954 capable of producing steam for the generation of electricity, propulsion, and space or process heat applications upon which the AEC has made a finding of practical value within the meaning of Section 102 of the Atomic Energy Act. (No reactors presently in the category)

LARGE NUCLEAR POWER PLANT A plant having a nuclear capacity greater than $350 \mathrm{Mw}(\mathrm{e})$ designed and constructed for operation as a central-station plant in a utility system Such plants over their lifetimes are expected to be economically competitive, or nearly so, with conventional plants in the geographic areas in which they are located (Part I, Sec 1A 1)

PROTOTYPE NUCLEAR POWER PLANT A nuclear power plant designed, constructed, and operated on a utılity system principally for the purpose of proving out economic and technical aspects of a future nuclear power plant of the same general type. The generation of significant quantities of electricity, mechanical power, or process heat is a function of such a plant. (Part I, Sec. 1A.2)

EXPERIMENTAL POWER PLANT A relatively complete plant designed, engineered, constructed, and operated to provide the technical basis for a similar nuclear power plant in a larger size. Design flexibility permits changes to prove out different aspects of reactor technology. Electricity or power generation may or may not be included as part of the plant. (Part I, Sec. 2A.1)

REACTOR EXPERIMENT A reactor in the research and development program, designed for the limited purpose of testing the technical feasibility of a reactor concept or some unique reactor feature or piece of equipment. A reactor experiment is built with the intention of making changes in fueling structure, assoc1ated components, or other components of the reactors (Part I, Sec. 2A.2). Advanced Reactor System Experiments (Part I, Sec. 2B) involve investigations of promising new concepts that have potential for application in several reactor development programs.

GENERAL IRRADIATION TEST REACIOR This category includes only those reactors having (1) a thermal output of $10,000 \mathrm{kw}$ or more, (2) test loops or experimental facilities within, or in proximity to, the core, and (3) the use of nuclear radiation for testing the life or performance of reactor components as its major function. (Part I, Sec. 3A, and Part IV, Sec. 2A)

SAFETY RESEARCH AND TEST REACTORS Includes reactors associated with a nuclear safety research or engineering-scale test program conducted for the purpose of developing basic design information or demonstrating safety characteristics of terrestrial, and aerospace nuclear reactor systems. (Part I, Sec. 3B)

SPECIAL TEST REACTORS This category includes reactors designed for special testing purposes (Part II, Sec. 3A, and Part III, Sec. 3)

RESEARCH REACTOR Any reactor whose nuclear radiations are used primarily as a research tool for basic or applied research regardless of operating power level. May include facilities for testing reactor materials. (Part I, Sec. 3C; Part II, Sec. 3B; and Part IV, Sec. 2B) 
TEACHING REACTOR Any reactor operated for the primary purpose of training in the operation and utilization of reactors and for instruction in reactor theory and performance. (Part I, Sec. 3D, and Part IV, Sec. 2C)

CRITICAL FACILITY A reactor capable of sustaining a nuclear chain reaction operating at extremely low power (a few watts) and designed to determine a critical mass, neutron-flux distribution, and other characteristics of a flexible arrangement of nuclear fuel, materials of construction, coolant, and other reactor components. Flund critical facilities are used to explore the critical masses of various concentrations of solutions in differing geometries. Metal critical assemblies are used to investigate the variations in heterogeneous cores. The tabulation of these facilities in Part $V$ excludes those that have been dismantled.

The abbreviated listings in the principal nuclear contractor column refer to the technical organization assigned primary responsibility for design and/or fabrication of the reactor system. The spelled-out forms for those abbreviations, as well as those for designers, shipbullders, and facility operators, are given in the table on page 4.

Start-up dates refer to the year of first criticality. Estımated dates for projects not yet in service, based on the best avallable information, are included. The dates for non-Commission projects are estımates announced by the sponsoring organizations.

Reactors are listed as being operable under the following circumstances.

1. Federal Government reactors - when criticality is achieved.

2. Non-Federal Government reactors in the United States- when an operating license is issued by the Commission.

3. Reactors for foreign locations - when criticality is achieved.

Reactors are listed as being bullt under the following circumstances:

1. Federal Government reactors - when ground is broken, components are ordered, or construction contract is awarded.

2. Non-Federal Government reactors in the United States - when the construction permit is issued by the Commission.

3. Reactors for foreign locations - when an application for an export license is received by the Commission or when reliable information is received relating to the fabrication of reactor components

Reactors are listed as being planned under the following circumstances.

1. Federal Government reactors - when publicly announced as a project planned for construction by the agency involved or the project is otherwise appropriately authorized.

2. Non-Federal Government reactors in the United States-when license application is received by the Commission or a public announcement that includes principal contractor and reactor type is made.

3. Reactors for foreign locations - when public announcement that includes principal contractor and reactor type is made or when the Commission receives information that a U.S. reactor manufacturer is proceeding with preconstruction design and development on the basis of a letter of intent.

The Statıstıcal Summary on page 3 does not include a breakdown for critical facilities. All other categories are summarized. Shutdown and dismantled reactors in these categories are included since such facilities have made significant contributions to reactor technology. 


\section{STATISTICAL SUMMARY}

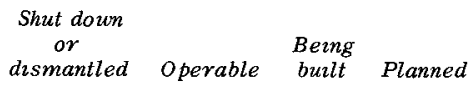

I CIVILIAN REACTORS (pages 5 to 13)

1 Power Reactors (pages 5 and 6)

A 1 Large Power Plants

A 2 Prototypes, Central-Station Plants

B Prototypes, Maritime Propulsion (Seagoing)

2 Experımental Reactor Systems (pages 6 to 9 )

A 1 Experimental Power Reactors (Generate Electricity)

A 2 Power-Reactor Experıments (Token Electrical Production, If Any)

B Advanced Reactor System Experiments

C Space Propulsion Experiments (ROVER)

D Auxilıary Power (SNAP)

3. Test, Research, and Unversity Reactors (pages 9 to 13)
A General Irradiation Test
B Safety Research and Test
C. General Research
D. University Research and Teaching

II MILITARY REACTORS (pages 13 to 16)

1 Defense Power-Reactor Applications (pages 13 and 14)

A Power Reactors, Remote Installations

B Propulsion Reactors (Naval)

2 Developmental Power Reactors (page 15)

A Power-Reactor Experiments and Prototypes

B 1 Naval Propulsion Reactor Prototypes

B 2 Aircraft-Propulsion Reactor Experiments

B 3 Missile-Propulsion Reactor Experiments (PLUTO)

3 Test and Research (pages 15 and 16)

A Test

B Research

ssmantled

perable bullt Planned

III PRODUCTION REACTORS (pages 16 and 17)

1 Materials Production (page 16)

2 Process Development (page 17)

3 Test (page 17)

IV REACTORS FOR EXPORT (pages 17 to 19)

1 Power Reactors (page 17)

A Central-Station Electric Power

B Propulsion

2 Test, Research, and Teaching Reactors (pages 18 and 19)

A General Irradiation Test

B General Research

C University Research and Teachıng

$\begin{array}{rrrr} & & 9 & 23 \\ 1 & 13 & 1 & 1 \\ & 1 & & \\ 4 & & & \\ & 3 & & 1 \\ 8 & 2 & 1 & \\ 4 & 1 & 1 & \\ 13 & & 5 & * \\ 7 & & 2 & \\ & & & \\ 1 & 4 & 1 & 1 \\ 6 & 4 & 2 & 1 \\ 13 & 45 & 4 & 2 \\ 3 & 43 & 5 & 1\end{array}$

$\begin{array}{lrrr}1 & 4 & 1 & \\ 2 & 79 & 36 & 8\end{array}$

RITICAL ASSEMBLY FACILITIES (pages 19 to 22)

1 Identification of Facilities (page 19)

2. Identification of Experiments and Studies (pages 20 to 22)

A. Civilian

B Military

*A number of engines and experimental reactors are expected to be tested 


\section{LIST OF CONTRACTORS, DESIGNERS, SHIPBUILDERS, AND FACILITY OPERATORS FOR WHICH ABBREVIATIONS APPEAR IN TABLES}

Allis-Chalmers Mfg. Co.

$\mathrm{ACF}$ Industries, Inc. (reactor activities absorbed by $\mathrm{AC}$ ) Aerojet-General Corporation

Aerojet-General Nucleonics formerly a subsidaary and now a Division of Aerojet-General Corporation

Atomics International, a Division of North American Aviation, Inc Alco Products, Inc. (reactor activities absorbed by $\mathrm{AC}$ )

AMF Atomics, Inc., a Division of American Machine \& Foundry Co. Argonne National Laboratory, operated by the University of Chicago

(naft nuclear Propulsion Department, General Electric Company

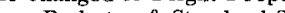

Bend1x Aviation Corp.

Shipbuilding Division, Bethlehem Steel Co (Now Quincy Division, General Dynamics Corp :

Bettis Atomic Power Laboratory operated by Westinghouse Electric Corporation

Bett1s

Blaw-Knox Blaw-Knox Co.

Brookhaven National Laboratory, operated by Associated Un1-

BNW

Comb.

Convair

Cook

Daystrom

DOD

du Pont

Ebasco

Electric Boat

FAST Ine

versities, Inc.

Battelle-Northwest a component of Battelle Memoraal Institut

Burns \& Roe, Inc.

Clinton Laboratory ot the Manhattan Engineer District

Combustion Engineering, Inc.

Nucledyne Co., a Division of Cook Electric Company

Curtiss-Wricht Corporation

Daystrom, Inc.

Department of Defense

The Dow Chemical Co., Rocky Flats Division

E. I. Du Pont de Nemours \& Company

Ebasco Services, Inc.

Electric Boat Division, General Dynamies Corp.

First Atomic Ship Transport Inc a subsidiary of Amesican Export Isbrandtsen Lines

Fluor

FW

The Fluor Corporation, Ltd.

oster Wheeler Corp.

Quincy Division Ceneral Dynamics Corp

Divelsor Coner

\begin{tabular}{||l} 
GE \\
GM \\
GNEC \\
GSA \\
HKF \\
Hughes \\
IC \\
INC \\
Ingalls \\
Kaman \\
KAPL \\
KE \\
LASL \\
Lockheed \\
Mare Island \\
Martin \\
Maxon \\
Met. Lab. \\
NASA \\
NBS \\
Newport News \\
NRL \\
ARTS \\
NYSC \\
ORNL \\
PNWL \\
Portsmouth \\
PPC \\
PRDC \\
P\&W \\
Sandıa \\
UCLRL \\
UNC \\
West. \\
\end{tabular}

General Electric Compan

General Motors Corp.

General Nuclear Engineering Corp. (became a division of Combustion Engineering, Inc., in 1964)

General Services Administration

H. K. Ferguson Co.

Hughes Aurcraft

Idaho Nuclear Corporation

Ingalls Shipbuilding Corp.

Kaman Nuclear, a Division of Kaman Aircraft Corp.

Knolls Atomic Power Laboratory, operated by General Electric Company

Calser Engineers, a Division of Henry J. Kaiser Co.

Las Alamos Scientific Laboratory, operated by the University of California

raft Corp.

San Franc1sco Bay Naval Shipyard, Mare Island

Martın-Marıetta Corp.

Metallurgical Laboratory of the Manhattan Engineer District

National Aeronautics and Space Admmistration

National Bureau of Standards

Newport News Shipbuilding \& Dry Dock Co.

Naval Research Laboratory

National Reactor Testing Station

Oak Rıdge National Laboratory, operated by Unıon Carbide Nuclear Company, a Division or Union Carbide Corporation Portsmouth Naval Shipyard

Phillips Petroleum Co.

Power Reactor Development Company

th \& Whitney Aircraft Division, United Aircraft Corp. Western Electric Co.

Unversity of Calıfornı Lawrence Radiation Laboratory

United Nuclear Corporation, Development Division

Westinghouse Electric Corporation 


\section{A. Central-Station Electric Power}

\begin{tabular}{|c|c|c|c|c|c|c|}
\hline \multirow[b]{2}{*}{ Name and/or owner } & \multirow[b]{2}{*}{ Location } & \multirow{2}{*}{$\begin{array}{l}\text { Principal } \\
\text { nuclear } \\
\text { contractor }\end{array}$} & \multirow[b]{2}{*}{ Type } & \multicolumn{2}{|c|}{ Power' } & \multirow[b]{2}{*}{$\begin{array}{l}\text { Start- } \\
\text { up }\end{array}$} \\
\hline & & & & $\begin{array}{c}\text { Plant, } \\
\text { net kw(e) }\end{array}$ & $\begin{array}{c}\text { Reactor, } \\
k w(t)\end{array}$ & \\
\hline
\end{tabular}

\section{A.1 Large Power Plants}

\section{BEING BUILT}

San Onofre Nuclear Generatıng Statıon (Southern Calıfornıa Edıson and

San Diego Gas and Electric Co.) ${ }^{2}$

Connecticut Yankee Atomic Power Plant (Connecticut Yankee Atomic

Power Co. $)^{2}$

Oyster Creek Nuclear Power Plant, Unit No. 1 (Jersey Central Power \& Light Co.)

Nine Mile Point Nuclear Station (Niagara Mohawk Power Corp)

Dresden Nuclear Power Station Unit No. 2 (Commonwealth Edison Co.)

Robert Emmett Ginna Nuclear Power Plant (Rochester Gas \& Electric Co ) Millstone Nuclear Power Station (Connecticut Light \& Power Co., Hartford

Electric Light Co, and Western Massachusetts Electric Co.)

Indian Point Station - Unit 2 (Consolidated Edison Co. of New York, Inc.)

Dresden Nuclear Power Station Unit 3 (Commonwealth Edıson Co.)

Malıbu Nuclear Plant Unit No. 1 (Los Angeles Department of Water and

$$
\text { Power })^{2}
$$

Turkey Point No 3 (Florida Power \& Light $\mathrm{CO}$ )

Turkey Point No. 4 (Florida Power \& Light Co)

Pilgrim Station (Boston Edıson Company)

H. B Robinson S E Plant Unit 2 (Carolina Power \& Light Co.)

Palısades Nuclear Power Station (Consumers Power Co. of Michigan)

Point Beach Nuclear Station (Wisconsin Michigan Power Co.)

Quad-Citıes Station Unit No. 1 (Commonwealth Edıson Co. \& Iowa-Illınoıs

Monticello Nuclear Generating Plant (Northern States Power Co)

Browns Ferry Nuclear Power Plant Unit No. 1 (TVA)

Browns Ferry Nuclear Power Plant Unit No. 2 (TVA)

Oconee Nuclear Station Unit No. 1 (Duke Power Co.)

Oconee Nuclear Station Unit No. 2 (Duke Power Co.)

Vermont Yankee Nuclear Generating Station (Vermont Yankee Nuclear Power Corp.)

Ft. Calhoun Plant (Omaha Public Power District)

Quad-Cities Station Unit No. 2 (Commonwealth Edıson Co. \& Iowa-Illınois Gas \& Electric Co.)

Peach Bottom Atomic Power Station Unit No. 2 (PEC, PSE\&GC, ACEC, $D P \& L C)$

Burlington Nuclear Generatıng Station (PSE\&GC, PEC, ACEC, DP\&LC)

Surry Power Station Unit No. 1 (Virgina Electric \& Power Co.)

Pacific Gas \& Electric Co.

Surry Power Station Unit No. 2 (Virginia Electrie \& Power Co.)

Niagara Mohawk Power Corp.

Metropolitan Edison Co.

Philadelphia Electric Company with PSE\&GC, ACEC, DP\&LC

San Clemente, Calif.

West.

Pressurized wate

$430,000 \quad 1,347,000$

Toms River, N. J.

Scriba, N. Y.

Morris, ill.

Ontario, N. Y.

Waterford, Conn

Indian Point, N. Y.

Morris, Ill.

Corral Canyon, Calıf.

Turkey Point, Fla.

Turkey Pount, Fla

Plymouth, Mass.

Hartsville, S. C.

Palısades Park, Mich.

Two Creeks, Wis.

Cordova, $\mathrm{nl}$.

Monticello, Minn.

Decatur, Ala.

Decatur, Ala.

Clemson, S. C.

Clemson, S. C.

Vernon, vt.

Ft. Calhoun, Nebr.

Cordova, Ill.

Peach Bottom, Pa.

Burlington, N. J.

Hog Island, Va.

Diablo Canyon, Calif.

Hog Island, Va.

Stıllwater, N. Y.

Pennsylvanı
GE

Pressurized water

Bolling water

Bouling water Bollung water Pressurized wate Bolling water

Pressurızed wate Bolling water

Pressurized water

West.

West.

West.

GE

West.

Comb.

West.

GE

GE

GE

GE
B\&W
$B \& W$

$\mathrm{GE}$

Comb.

Com

GE

GE

West.

West.

West.

West.

GE

$B \& W$

Pressurized water Pressurized water Bolling water Pressurized water Pressurized water Pressurized water

Bolling water Bolling water

Bolling water

Boiling water

Pressurized water

Pressurızed water

Bollung water

Pressurized water

Bolling water

Bollung water

Pressurized water Pressurized water Pressurized water Pressurızed water Bolling water

Pressurized water

Boling water
$1,064,500 \quad 3,293,000$

Footnotes appear on pare 23 and 24

$462,000 \quad 1,473,000$

$515,000 \quad 1,600,000$

$500,000 \quad 1,538,000$

$715,000 \quad 2,255,000$

$420,000 \quad 1,300,000$

$549,200 \quad 1,727,000$

$872,900 \quad 2,758,000$

$715,000 \quad 2,225,000$

$462,000 \quad 1,727,000$

$721,500 \quad 2,300,000$

$721,500 \quad 2,300,000$

549,000

$663,000 \quad 2,094,000$

$700,000 \quad 2,200,000$

$454,600 \quad 1,396,000$

$715,000 \quad 2,300,000$

$471,700 \quad 1,469,000$

$1,064,500 \quad 3,293,000$

$839,000 \quad 2,452,000$

$839,000 \quad 2,452,000$

$513,900 \quad 1,593,000$

450,000

$715,000-2,300,000$

$1,065,000 \quad 3,294,000$

1967

1967

1967

1968

968

969

1969

1969

1970

1970

1971

1970

1970

1970

1970

1970

1971

1971

1972
1970

1970

1971

$993,000 \quad 3,083,000$

$783,000 \quad 2,441,000$

$1,060,000$

$783,000 \quad 2,441,000$

750,000

839,000

$1,065,000 \quad 3,294,000$ 


\section{A. Central-Station Electric Power}

\section{Name and/or oumer}

Location

Principal

nuclear

Power $^{1}$

A. 2 Power-Plant Prototypes

SHUT DOWN OR DISMANTLED

Hallam Nuclear Power Facility, Sheldon Station (AEC and Consumers Public Hallam, Nebr. Power District ${ }^{2}$

$$
\text { OPERABLE }
$$

Shippingport Atomic Power Station (AEC and Duquesne Light Co. ${ }^{4}$

Dresden Nuclear Power Station (Commonwealth Edıson Co.)

Yankee Nuclear Power Station (Yankee Atomic Electric $\mathrm{Co}$.) ${ }^{2}$

Big Rock Point Nuclear Power Plant (Consumers Power Co.) ${ }^{2}$

Elk River Reactor (AEC and Rural Cooperative Power Association) ${ }^{2,5}$

Indian Point Station - Unit 1 (Consolidated Edison Co. of New York, Inc.) ${ }^{6}$

Carolınas-Virginia Tube Reactor (Carolınas-Virginaa Nuclear Power

Associates, Inc.) ${ }^{2}$ ?

Enrico Fermi Atomic Power Plant (Power Reactor Development Co.) ${ }^{2}$

Humboldt Bay Power Plant, Unit No. 3 (Pacific Gas and Electric Co.

Piqua Nuclear Power Facility (AEC and City of Plqua)

Bolling Reactor Nuclear Superheat Project (AEC and Puerto Rico Water Resources Author ity)

Pathfinder Atomic Power Plant (Northern States Power Co.) $)^{2}$

Peach Bottom Atomic Power Station Unit No. 1-H rGR (Philadelphia Electric Co.) $)^{2}$

BEING BUILT

La Crosse Bollng Water Reactor (AEC and Dauryland Power Cooperative) PLANNED

Fort St. Vrain Nuclear Generating Station (Public Service Co

of Colorado $)^{2}$

Shippingport, $\mathrm{Pa}$

Morris, Ill.

Rowe, Mass.

Big Rock Point, Mich.

Ind Rer

Parr, S. C.

Lagoona Beach, Mich. Eureka, Calif. Piqua, Oh10

Punta Higuera, P. R.

Sioux Falls, S. Dak.

Peach Bottom, Pa

Genoa, Wis.

Platteville, Colo
AI

West.

West.

GE

West.

PRDC

GE

AI

Comb.

AC

GDC

(1)

AC

GDC
Sodium graphite

Pressurized water

Bolling water

Pressurızed water

Bolling water

Bollng water

Pressurized water

Pressure tube, heavy

Sodium cooled, fast

Bolling water

Organze cooled and

moderated

Boiling water, integra

nuclear superheat

Bolling water, nuclear

superheat

Gas cooled, graphite
moderated

Boilnng water

Gas cooled, graphite

moderated net kw(e)

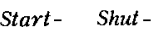

up down

\section{B. Propulsion (Maritime Seagoing Prototypes)}

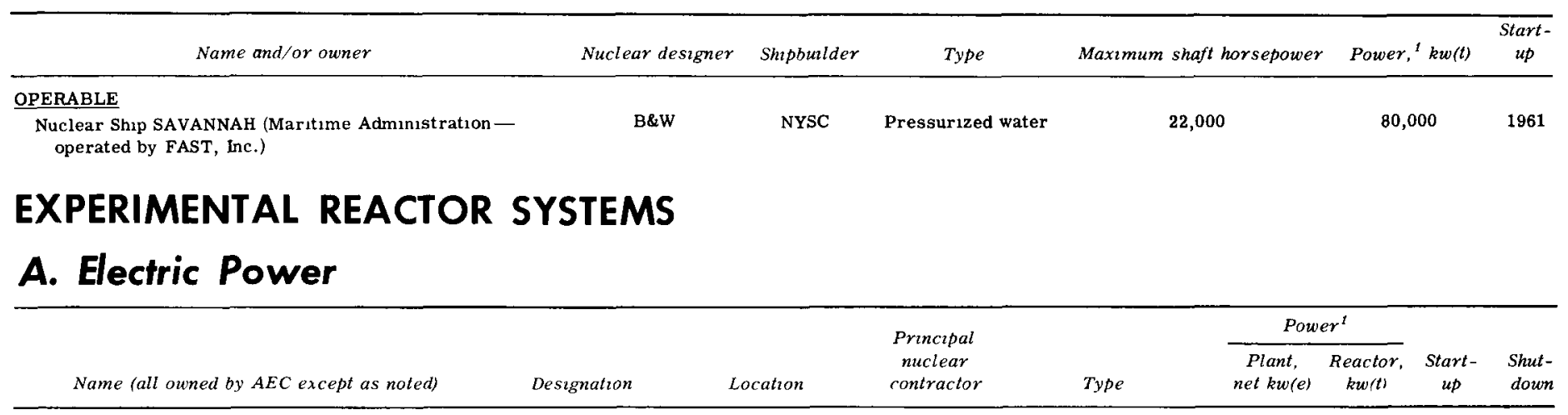

A.1 Experimental Reactors 
Experımental Gas-Cooled Reactor (AEC and

Tennessee Valley Authority) ${ }^{8}$

Sodium Reactor Experiment (AEC and Souther

Californa Edison $\mathrm{Co}.)^{10}$

\section{OPERABLE}

Experime Bolling Water Reactor

Saxton Nuclear Experimental Reactor Project

(Saxton Nuclear Experimental Corp.)

actor No. 2

PLANNED

Small Unified Reactor Facility with Systems

for Isotopes, Desalting, and Electricity (NYASDA) $^{12}$

\section{A.2 Reactor Experiments}

\section{SHUT DOWN OR DISMANTLED}

Bollung Reactor Experiment No. 1
Homogeneous Reactor Experiment No. 1

Boilıng Reactor Experiments ${ }^{13}$

Homogeneous Reactor Experıment No. 2

Organic Moderated Reactor Experıment ${ }^{14}$

Experımental Breeder Reactor No. $1^{15}$

Heavy Water Components Test Reactor

Experımental Organıc Cooled Reactor ${ }^{16}$

OPERABLE

Plutomum Recycle Test Reactor

ESADA Vallecitos Experimental Superheat Reactor (Empire States Atomic Development Associates and General Electric Company) BEING BUILT

Southwest Experimental Fast Oxide Reactor (Southwest Atomic Energy Associates)

$\begin{array}{ll}\begin{array}{l}\text { ANL } \\ \text { West. }\end{array} & \begin{array}{l}\text { Boiling water } \\ \text { Pressurıea water }\end{array} \\ \text { ANL } & \text { Sodium cooled, fast } \\ \text { AMF } & \begin{array}{l}\text { Pressurized water, } \\ \text { Atomics }\end{array} \\ \end{array}$

\section{4,000}

$100,000 \quad 1956$

BORAX HRE -1

BORAX-2,

3,4
HRE-2

OMRE

EBR-1

HWCTR

EOCR

PRTR

EVESR

SE FOR
NRTS, Idaho Oak Ridge, Tenn

NRTS, Idaho

Oak Ridge, Tenn.

NRTS, Idaho

NRTS, Idaho

Savannah River

Laboratory,
Alken, S. C.

NRTS, Idaho

Richland, Wash.

Pleasanton, Calıf.

Strickler, Ark.
Aqueous homogeneous

solution $\left(\mathrm{UO}_{2} \mathrm{SO}_{4}\right)$

Boiling water

ORNL Aqueous homogeneous

solution $\left(\mathrm{UO}_{2} \mathrm{SO}_{4}\right)$

Organic cooled and

moderated

ANL Sodium cooled, fas

du Pont Pressurized heavy water

Fluor-AI Organic cooled and moderated

GE

Pressure tube, heavy water moderated and cooled

GE Light water nod
superheate

GE Sodium cooled, fast
No elec. $\quad 1,400 \quad 195$

$140 \quad 1,000 \quad 1952$

$2,400 \quad 15,500 \quad 1954$

300

$5,200 \quad 1957$

No elec. $\quad 16,000 \quad 1957$

$150 \quad 1,400 \quad 1951$

$\begin{array}{rrrr}\text { No elec. } \quad 61,000 & 1962 & 1964\end{array}$

No elec. $\quad 40,000$

No elec. $\quad 120,000 \quad 1960$

No elec. $\quad 17,000 \quad 1963$

No elec. $\quad 20,000 \quad 1968$

\section{B. Advanced Reactor System Experiments}

\section{SHUT DOWN OR DISMANTLED}

Los Alamos Power Reactor Experıment No. 1

Los Alamos Power Reactor Experiment No 2

Los Alamos Molten Plutonum Reactor Experiment

Experimental Beryllium Oxıde Reactor ${ }^{17}$

OPERABLE

Molten Salt Reactor Experiment

BEING BUILT

Ultra High Temperature Reactor Experıment

\section{LAPRE-2}

Los Alamos, N. Mex.

LAMPRE - 1

Los Alamos, N. Mex.

EBOR

NRTS, Idaho

MSRE

Oak Ridge, Tenn.

UHTREX
LASL

LASL

LASL

GDC

ORNL
Aqueous homogeneous (phosphoric acid) Aqueous homogeneous (phosphoric acid)

Fast molten plutonium
fueled, sodium cooled Gas cooled, BeO moderated

No elec. $\quad 2,000 \quad 1956$ No elec. $\quad 1,000 \quad 1959$ No elec. $\quad 1,000 \quad 1961$ No elec. $\quad 10,000$ Single regıon graphite moderated

No. elec. $\quad 10,000 \quad 1965$ Helium cooled 


\section{Space Propulsion Experiments (Rover) ${ }^{18}$}

\begin{tabular}{|c|c|c|c|c|c|c|}
\hline Name (all oumed bi $A E C$ a xecpt as noted) & Designation & Location & $\begin{array}{l}\text { Principal } \\
\text { nuclear } \\
\text { contractor }\end{array}$ & Type & $\begin{array}{c}\text { Reactor pouts, } \\
\text { kut (t) }\end{array}$ & Operated \\
\hline \multicolumn{7}{|l|}{ SHUT DOWN OR DISMANTLED } \\
\hline Nuclear Rocket Reactor Experıment & KIWI-A & NRDS, Nev. & LASL & $\begin{array}{l}\text { Open cycle, hydrogen } \\
\text { gas cooled }\end{array}$ & 70,000 & 1959 \\
\hline Nuclear Rocket Reactor Experiment & $\begin{array}{l}\text { KIWI-A } \\
\text { Prime }\end{array}$ & NRDS, Nev. & LASL & $\begin{array}{l}\text { Open cycle, hydrogen } \\
\text { gas cooled }\end{array}$ & 85,000 & 1960 \\
\hline Nuclear Rocket Reactor Experıment & KIWI-A3 & NRDS, Nev. & LASL & $\begin{array}{l}\text { Open cycle, hydrogen } \\
\text { gas cooled }\end{array}$ & 100,000 & 1960 \\
\hline Nuclear Rocket Reactor Experıment & KTWI-B1A & NRDS, Nev. & LASL & $\begin{array}{l}\text { Open cycle, hydrogen } \\
\text { gas cooled }\end{array}$ & 300,000 & 1961 \\
\hline Nuclear Rocket Reactor Experıment & KIWI-B1B & NRDS, Nev. & LASL & $\begin{array}{l}\text { Open cycle, liquid } \\
\text { hydrogen }\end{array}$ & 900,000 & 1962 \\
\hline Nuclear Rocket Reactor Experıment & KIWI-B4A & NRDS, Nev. & LASL & $\begin{array}{l}\text { Open cycle, liquid } \\
\text { hydrogen }\end{array}$ & 500,000 & 1962 \\
\hline Nuclear Rocket Reactor Experıment & KIWI-B4D & NRDS, Nev. & LASL & $\begin{array}{l}\text { Open cycle, liquid } \\
\text { hydrogen }\end{array}$ & $1,000,000$ & 1964 \\
\hline Nuclear Rocket Reactor Experiment & $\mathrm{KIWI}-\mathrm{B} 4 \mathrm{E}$ & NRDS, Nev. & LASL & $\begin{array}{l}\text { Open cycle, liquid } \\
\text { hydrogen }\end{array}$ & 950,000 & 1964 \\
\hline $\begin{array}{l}\text { Nuclear Rocket Engine Reactor Experiment } \\
\text { (NERVA) }\end{array}$ & NRX-A2 & NRDS, Nev. & AG-West. & $\begin{array}{l}\text { Open cycle, liquid } \\
\text { hydrogen }\end{array}$ & $1,100,000$ & 1964 \\
\hline $\begin{array}{l}\text { Nuclear Rocket Engine Reactor Experiment } \\
\text { (NERVA) }\end{array}$ & NRX $-A 3$ & NRDS, Nev & AG-West. & $\begin{array}{l}\text { Open cycle, liquid } \\
\text { hydrogen }\end{array}$ & $1,120,000$ & 1965 \\
\hline Nuclear Rocket Reactor Experıment & Phoebus 1A & NRDS, Nev. & LASL & $\begin{array}{l}\text { Open cycle, liquid } \\
\text { hydrogen }\end{array}$ & $1,070,000$ & 1965 \\
\hline $\begin{array}{l}\text { Nuclear Rocket Reactor Engıne System Test } \\
\text { (NERVA) }\end{array}$ & $\begin{array}{l}\text { NRX }-\mathrm{A} 4 / \\
\text { EST }\end{array}$ & NRDS, Nev & AG-West & $\begin{array}{l}\text { Open cycle, liquid } \\
\text { hydrogen }\end{array}$ & $1,190,000$ & 1966 \\
\hline $\begin{array}{l}\text { Nuclear Rocket Engine Reactor Experiment } \\
\text { (NERVA) }\end{array}$ & NRX $-\mathrm{A} 5$ & NRDS, Nev & AG-West & $\begin{array}{l}\text { Open cycle, liquid } \\
\text { hydrogen }\end{array}$ & $1,100,000$ & 1966 \\
\hline \multicolumn{7}{|l|}{ BEING BUILT } \\
\hline Nuclear Rocket Reactor Experıment & Phoebus 1B & NRDS, Nev & LASL & $\begin{array}{l}\text { Open cycle, liquid } \\
\text { hydrogen }\end{array}$ & & 1967 \\
\hline Nuc lear Rocket Reactor Experıment & Phoebus 2A & NRDS, Nev & LASL & $\begin{array}{l}\text { Open cycle, liquid } \\
\text { hydrogen }\end{array}$ & & 1967 \\
\hline $\begin{array}{l}\text { Nuclear Rocket Engine Reactor Experiment } \\
\text { (NERVA) }\end{array}$ & NRX $-A 6$ & NRDS, Nev & $A G-$ West & $\begin{array}{l}\text { Open cycle, liquid } \\
\text { hydrogen }\end{array}$ & & 1967 \\
\hline Ground Experımental Engine Experiment & $X E-1$ & NRDS, Nev & $\mathrm{AG}-$ West & $\begin{array}{l}\text { Open cycle, liquid } \\
\text { hydrogen }\end{array}$ & & 1967 \\
\hline Nuclear Rocket Reactor Experiment & $\begin{array}{l}\text { Phoebus 1C } \\
\text { series }\end{array}$ & NRDS, Nev & LASL & $\begin{array}{l}\text { Open cycle, liquid } \\
\text { hydrogen }\end{array}$ & & \\
\hline \multicolumn{7}{|l|}{ PLANNED } \\
\hline Ground Experımental Engine Test & $X E$ series & NRDS, Nev & $\mathrm{AG}-$ West & $\begin{array}{l}\text { Open cycle, liquid } \\
\text { hydrogen }\end{array}$ & & \\
\hline Nuc lear Rocket Reactor Experiments & $\begin{array}{c}\text { Phoebus } 2 \\
\text { series }\end{array}$ & NRDS, Nev & LASL & $\begin{array}{l}\text { Open cycle, liquid } \\
\text { hydrogen }\end{array}$ & & \\
\hline
\end{tabular}

\section{Auxiliary Power (SNAP)}




\section{TEST, RESEARCH, AND UNIVERSITY REACTORS}

\section{A. General Irradiation Test}

\begin{tabular}{|c|c|c|c|c|c|c|c|c|}
\hline Name and/or owner & Designation & Location & $\begin{array}{c}\text { Princtpal } \\
\text { nuclear coniractor }\end{array}$ & Operator & Type & $\begin{array}{c}\text { Power, } \\
k w(t)\end{array}$ & $\begin{array}{l}\text { Start- } \\
u p\end{array}$ & $\begin{array}{l}\text { Shut - } \\
\text { doun }\end{array}$ \\
\hline \multicolumn{9}{|l|}{ SHUT DOWN OR DISMANTLED } \\
\hline $\begin{array}{l}\text { Westinghouse Testing Reactor } \\
\text { OPERABLE }\end{array}$ & WTR & Waltz Mill, Pa. & Owner & Owner & Tank & 60,000 & 1959 & 1962 \\
\hline Materials Testıng Reactor $(\mathrm{AEC})^{20}$ & MTR & NRTS, Idaho & ORNL-ANL-Blaw-Knox & INC & Tank & 40,000 & 1952 & \\
\hline Engmeering Test Reactor (AEC) & ETR & NRTS, Idaho & $\mathrm{KE}-\mathrm{GE}$ & INC & Tank & 175,000 & 1957 & \\
\hline General Electric Testing Reactor & GETR & Pleasanton, Calif. & Owner & Owner & Tank & 50,000 & 1958 & \\
\hline Plum Brook Reactor Facility (NASA) & NASA -TR & Sandusky, Ohro & NASA & NASA & Tank & 60,000 & 1961 & \\
\hline \multicolumn{9}{|l|}{ BEING BUILT } \\
\hline Advanced Test Reactor (AEC) & ATR & NRTS, Idaho & Ebasco-B\&W & INC & Tank & 250,000 & 1967 & \\
\hline PLANNED & & & & & Sodium & & & \\
\hline Fast Flux Test Facility & FFTF & & BNW & & cooled & 400,000 & 1972 & \\
\hline
\end{tabular}

\section{B. Safety Research and Test}

\begin{tabular}{|c|c|c|c|c|c|c|c|}
\hline Name (all ouned by $A F C$ ) & Designation & Location & $\begin{array}{c}\text { Principal } \\
\text { nuclear contractor }\end{array}$ & Type & $\begin{array}{c}\text { Pouer, } \\
\text { kw }(t)\end{array}$ & $\begin{array}{l}\text { Start- } \\
u p\end{array}$ & $\begin{array}{l}\text { Shut - } \\
\text { down }\end{array}$ \\
\hline \multicolumn{8}{|l|}{ SHUT DOWN OR DISMANTLED } \\
\hline SNAP 10A Transient Test No $3^{21}$ & SNAPTRAN-3 & NRTS, Idaho & PPC-AI & $\begin{array}{c}\mathrm{H}_{2} \mathrm{O} \text {-reflected } \\
\text { SNAP 10A }\end{array}$ & Transient & 1964 & 1964 \\
\hline Special Power Excursion Reactor Test No. $1^{22}$ & SPERT-1 & NRTS, Idaho & PPC & Open tank & Transient & 1955 & 1964 \\
\hline K1w1-Transient Test Reactor & $K_{1} w_{1}-T N T$ & NRDS, Nev. & LASL & $\mathrm{KIWI} / \mathrm{NERVA}$ & Transient & 1965 & 1965 \\
\hline Special Power Excursion Reactor Test No. $2^{23}$ & SPERT - 2 & NRTS, Idaho & PPC & $\begin{array}{l}\text { Pressurized } \\
\text { water }\end{array}$ & Transient & 1960 & 1965 \\
\hline SNAP 10A Transient Test No $2^{21}$ & SNAPTRAN - 2 & NRTS, Idaho & AI - PPC & $\begin{array}{l}\text { Be-reflected } \\
\text { SNAP 10A }\end{array}$ & Transient & 1965 & 1966 \\
\hline \multicolumn{8}{|l|}{ OPERABLE } \\
\hline Kinetic Experiment on Water Bollers & KEWB & Santa Susana, Caluf. & AI & Homogeneous & Transient & 1956 & \\
\hline Special Power Excursion Reactor Test No. 3 & SPERT - 3 & NRTS, Idaho & PPC & $\begin{array}{l}\text { Pressurized } \\
\text { water }\end{array}$ & Transient & 1958 & \\
\hline Special Power Excursion Reactor Test No. 4 & SPERT - 4 & NRTS, Idaho & PPC & Pool & Transient & 1962 & \\
\hline Transient Reactor Test & TREAT & NRTS, Idaho & ANL & Graphite & Transient & 1959 & \\
\hline Reactivity Accident Test & REACT & NRTS, Idaho & PPC & & Transient & & \\
\hline
\end{tabular}




\section{TEST, RESEARCH, AND UNIVERSITY REACTORS C. General Research}

\begin{tabular}{|c|c|c|c|c|c|c|c|}
\hline Name and/or owner & Designation & Location & $\begin{array}{l}\text { Principal } \\
\text { nuclear contractor }\end{array}$ & Type & $\begin{array}{c}\text { Power, } \\
\quad k w(t)\end{array}$ & $\begin{array}{l}\text { Start- } \\
\text { up }\end{array}$ & $\begin{array}{l}\text { Shut }- \\
\text { down }\end{array}$ \\
\hline \multicolumn{8}{|l|}{ SHUT DOWN OR DISMANTLED } \\
\hline $\begin{array}{l}\text { Chicago P1le 1, rebult as CP -2 (Manhattan } \\
\text { Engineer District-AEC) })^{24}\end{array}$ & $\mathrm{CP}-1$ & Chicago, Ill. & Met. Lab. & Graphite & $0.2-2$ & 1942 & 1954 \\
\hline Oak Ridge Graphite Reactor (AEC) & $x-10$ & Oak Ridge, Tenn. & CL & Graphite & 3,800 & 1943 & 1963 \\
\hline $\begin{array}{l}\text { Argonne } \mathrm{CP}-3 \text {, rebult as } \mathrm{CP}-3^{\prime} \\
\text { (Manhattan Engmeer District-AEC) }\end{array}$ & $\mathrm{CP}-3^{\prime}$ & Palos Park, Ill. & Met. Lab. & Heavy water & 300 & 1944 & 1954 \\
\hline Los Alamos Water Boller (AEC) & HYPO & Los Alamos, N. Mex. & LASL & Homogeneous & 5.5 & 1944 & 1950 \\
\hline Los Alamos Fast Reactor (AEC) & Clementine & Los Alamos, N. Mex. & LASL & $\begin{array}{l}\text { Fast, plutonium fuel, } \\
\text { mercury cooled }\end{array}$ & 25 & 1946 & 1953 \\
\hline Livermore Water Boller (AEC) & LIWB & Livermore, Calif. & AI & Homogeneous & 0.5 & 1953 & 1961 \\
\hline Atomics International & $\mathrm{L}-47$ & Canoga Park, Calif. & AI & Homogeneous & Neglig. & 1957 & 1958 \\
\hline Amerıcan Radiator \& Standard Sanitary Corp. ${ }^{25}$ & UTR-1 & Mountain View, Calıf. & ARSS & Graphite/water & Neglig. & 1958 & 1960 \\
\hline Lockheed Aircraft Corp. & & Dawsonville, Ga. & Lockheed & Pool & Neglig. & 1960 & 1960 \\
\hline $\begin{array}{l}\text { General Dynamics Corp. (World Agricultural } \\
\text { Fair - U. S. Exhıbit Reactor) }\end{array}$ & TRIGA - Mk II & San Diego, Calıf. & GDC & $\mathrm{U}-\mathrm{Zr}$ hydride, $\operatorname{tank}$ & 50 & 1960 & 1960 \\
\hline $\begin{array}{l}\text { UTR Test Reactor (American Radiator \& } \\
\text { Standard Sanitary Corp.) }\end{array}$ & & Mountain View, Calıf. & Owner & Graphite/water & Neglig. & 1961 & 1963 \\
\hline $\begin{array}{l}\text { Louisiana State University Nuclear Science } \\
\text { Center (Sandia Nuclear Assembly for } \\
\text { Reactor Experiments) }\end{array}$ & SNARE & Baton Rouge, La & Sandıa & Pool & 1 & 1965 & 1966 \\
\hline Aerojet-General Corporation & AGN-201P-103 & San Ramon, Calıf. & AGN & Homog. solıd & Neglig. & 1957 & 1966 \\
\hline \multicolumn{8}{|l|}{ OPERABLE } \\
\hline Brookhaven Research Reactor (AEC) & BGRR & Upton, N. Y. & HKF & Graphite & 20,000 & 1950 & \\
\hline Bulk Shrelding Reactor $(\mathrm{AEC})^{28}$ & BSR-1-BSR-2 & Oak Ridge, Tenn. & ORNL & Pool & 2,000 & 1950 & \\
\hline Los Alamos Water Boller (AEC) & SUPO & Los Alamos, N. Mex. & LASL & Homogeneous & 25 & 1950 & \\
\hline Low Intensity Test Reactor $(\mathrm{AEC})^{29}$ & LITR & Oak Ridge, Tenn. & ORNL & Tank & 3,000 & 1950 & \\
\hline $\begin{array}{l}\text { North American Aviation Water Boiler } \\
\text { Neutron Source (AEC) }\end{array}$ & AE-6(WBNS) & Santa Susana, Calıf. & AI & Homogeneous & 3 & 1952 & \\
\hline Argonne Research Reactor (AEC) & $\mathrm{CP}-5$ & Argonne, Ill. & ANL & Heavy water & 5,000 & 1954 & \\
\hline Battelle Memorial Institute & BRR & West Jefferson, Ohıo & AMF & Pool & 2,000 & 1956 & \\
\hline $\begin{array}{l}\text { Illınois Institute of Technology Research } \\
\text { Institute (Armour Research Foundation) }\end{array}$ & $\operatorname{ARR}(L-54)$ & Chıcago, Ill. & AI & Homogeneous & 75 & 1956 & \\
\hline Omega West Reactor (AEC) & OWR & Los Alamos, N. Mex. & LASL & Tank & 8,000 & 1956 & \\
\hline Argonne National Laboratory (AEC) & $A G N-201-108$ & Argonne, Ill. & AGN & Homog. solıd & Neglig. & 1957 & \\
\hline $\begin{array}{l}\text { Argonne Nuclear Assembly for University } \\
\text { Training (AEC) }\end{array}$ & $\begin{array}{l}\text { Argonaut } \\
\quad(\mathrm{CP}-11)\end{array}$ & Argonne, Ill. & ANL & Graphite/water & 10 & 1957 & \\
\hline General Electric Nuclear Test Reactor & NTR & Pleasanton, Calif. & $\mathrm{GE}$ & Light water & 30 & 1957 & \\
\hline Livermore Pool Type Reactor (AEC) & LPTR & Livermore, Calıf. & FW & Tank & 2,000 & 1957 & \\
\hline Atomics International & $\mathrm{L}-77$ & Canoga Park, Calif. & AI & Homogeneous & Neglig. & 1958 & \\
\hline Babcock \& Wilcox Lynchburg Pool Reactor & LPR & Lynchburg, Va. & Owner & Pool & 1,000 & 1958 & \\
\hline Brookhaven Neutron Source Reactor 1 (AEC) & SCHIZO & Upton, N. Y. & BNL & Tank & 100 & 1958 & \\
\hline General Dynamics Corp. & TRIGA-Mk I & La Jolla, Calıf. & GDC & $\mathrm{U}-\mathrm{Zr}$ hydride, tank & 250 & 1958 & \\
\hline $\begin{array}{l}\text { Industrial Reactor Laboratories, Inc. } \\
\text { (nnerated he Columbis Invercitu) }\end{array}$ & & Plainsboro, N. J. & AMF & Pool & 5,000 & 1958 & \\
\hline
\end{tabular}


AFSR

Omaha Veterans Administration Hospital Puerto Rico Nuclear Center (AEC)

General Dynamics Corp. TRIGA Pulsing Reactor ${ }^{32}$

TRIGA-Mk I

ANL

Omaha, Nebr.

TRIGA-Mk F

Mayaguez, P. R.

La Jolla, Calif.

Fast

$\mathrm{U}-\mathrm{Zr}$ hydrıde, tank

1

Puerto Rico Nuclear Center (AEC)

Sandia Pulsed Reactor (AEC)

Unıon Carbide Corp., Nuclear Division

Argonne Low Power Research Reactor $(\mathrm{AEC})^{33}$

Health Physics Research Reactor $(\mathrm{AEC})^{34}$

Sandia Engineering Reactor (AEC)

NASA Mock-up Reactor

Northrop Corporation (Space Radiation

$$
\text { Laboratory) }
$$

USAEC European Exhibit Program ${ }^{35}$

Babcock \& Wilcox Nuclear Development Center Test Reactor

Biological Research Reactor (AEC)

Rhode Island Nuclear Science Center

Shield Test and Irradiation Reactor

$(\mathrm{AEC})^{36}$

AGN Industrial Reactor

Ames Laboratory Research Reactor

(AEC)

Brookhaven Neutron Source Reactor No, 2

$$
\text { (AEC) }
$$

Brookhaven High Flux Beam Research Reactor (AEC)

High Flux Isotope Reactor (AEC)

General Dynamics Corp. Thermionic

Research TRIGA Reactor

\section{BEING BUILT}

National Bureau of Standards

High Temperature Lattıce Test Reactor (AEC)

Sandia Pulsed Reactor (AEC)

Annular Core Pulsed Reactor (AEC)

Dow Chemical $\mathrm{Co}$

Fast Neutron Source Reactor

\section{PLANNED}

Argonne Advanced Research Reactor (AEC)

Radiatıon Effects Reactor-II

U. S Geological Survey Laboratory
Mayagúez, P. R.

SPR

UCC - ND

Juggernaut

Sandia Base, N. Mex.

Sterling Forest, N. Y.

Argonne, Ill.

HPRR

Oak Ridge, Tenn.

SER

MUR

Sandia Base, N. Mex.

BAWTR

Lynchburg, Va.

JANUS

Argonne, Ill.

STIR

AGNIR

ALRR

PHRENIC

HFBR

Ft. Kearney, R. I.

Santa Susana, Calif.

San Ramon, Caluf.

Ames, Iowa

Upton, N. Y.

Upton, N. Y.

HFIR

TRIGA-Mk III

Oak Ridge, Tenn.

La Jolla, Calıf.

NBSR

HTLTR

Gaithersburg, Md.

Richland, Wash.

SPR-II

ACPR

TRIGA-Mk I

Sandia Base, N. Mex.

Sandia Base, N. Mex.

Midland, Mich.

Upton, N. Y.

Argonne, Ill.

Dawsonville, Ga.

RER-II

Denver, Colo
TRIGA-Mk F Hawthorne, Calıf.

$\mathrm{U}-\mathrm{Zr}$ hydrıde, tank
GDC

Homogeneous

U-Zr hydrıde, tank

Owner

AMF

Sandia

Pool

Prompt burst

Pool

Graphite/water

ANL

ORNL

Sand1a

Lockheed

GDC

Lockheed

Owner

ANL

GE

AGN

BNL

BNL

Fast burst

Tank

Light water, pool

$\mathrm{U}-\mathrm{Zr}$ hydride, tank

Pool

Pool

Tank

Pool

Pool

Pool

Heavy water

Tank

Heavy water

Tank flux trap

Owner

NBS-B\&R

BNW

Heavy water

Graphite

Prompt burst

$\mathrm{U}-\mathrm{Zr}$ hydride, pool

$\mathrm{U}-\mathrm{Zr}$ hydride, tank

Fast

1,500

1959

959

1960

Sandia

GDC

BNL

ANL

Light water, tank

1970 
3. TEST, RESEARCH, AND UNIVERSITY REACTORS

\section{University Research and Teaching*}

\begin{tabular}{|c|c|c|c|c|c|c|c|}
\hline Name and/or owner & Designation & Location & $\begin{array}{l}\text { Principal } \\
\text { nuclear contractor }\end{array}$ & Type & $\begin{array}{l}\text { Power, } \\
\text { kw }(t)\end{array}$ & $\begin{array}{l}\text { Start- } \\
u p\end{array}$ & $\begin{array}{l}\text { Shut - } \\
\text { down }\end{array}$ \\
\hline \multicolumn{8}{|l|}{ SHUT DOWN OR DISMANTLED } \\
\hline Pennsylvanıa State Unıversity & & University Park, Pa. & Owner & Pool & 200 & 1955 & 1965 \\
\hline Willam Marsh Rice University & AGN-211-101 & Houston, Tex. & AGN & Homog. solıd, pool & Neglig. & 1959 & 1965 \\
\hline $\begin{array}{l}\text { The Curtiss-Wright Nuclear Research } \\
\text { Laboratory of the Pennsylvania State } \\
\text { University }\end{array}$ & & Quehanna, Pa. & Owner & Pool & 1,000 & 1958 & 1966 \\
\hline \multicolumn{8}{|l|}{ OPERABLE } \\
\hline Texas A\&M Unıversity & AGN-201-106 & College Station, Tex. & AGN & Homog. solid & Neglig. & 1957 & \\
\hline Catholuc University of America & AGN-201-101 & Washıngton, D. C. & AGN & Homog. solid & Neglig. & 1957 & \\
\hline Colorado State University & AGN-201-109 & Ft. Colluns, Colo. & AGN & Homog. solid & Neglig. & 1957 & \\
\hline $\begin{array}{l}\text { Oklahoma State University of Agriculture } \\
\text { and Applied Science }\end{array}$ & AGN-201-102 & Stıllwater, Okla. & AGN & Homog. solıd & Neglig. & 1957 & \\
\hline University of Akron & AGN-201-104 & Akron, Oh1o & AGN & Homog. solid & Neglig. & 1957 & \\
\hline University of New Mexico ${ }^{37}$ & AGN-201-112 & Albuquerque, N. Mex. & AGN & Homog. solid & Neglig. & 1957 & \\
\hline $\begin{array}{l}\text { University of Michigan (Ford Nuclear } \\
\text { Reactor) }\end{array}$ & & Ann Arbor, Mich. & B\&W & Pool & 2,000 & 1957 & \\
\hline University of Utah & AGN-201-107 & Salt Lake City, Utah & AGN & Homog. solıd & Neglig. & 1957 & \\
\hline Massachusetts Institute of Technology & MITR & Cambridge, Mass. & $\mathrm{ACF}$ & Heavy water & 5,000 & 1958 & \\
\hline Oregon State Unıversity & AGN-201-114 & Corvallıs, Oreg. & AGN & Homog, solid & Neglig. & 1958 & \\
\hline Unıversity of Arizona & TRIGA-Mk I & Tucson, Arı. & GDC & $\mathrm{U}-\mathrm{Zr}$ hydrıde, tank & 100 & 1958 & \\
\hline Unıversity of Delaware & AGN-201-113 & Newark, Del. & AGN & Homog. solid & Neglig. & 1958 & \\
\hline University of Oklahoma & AGN-211-102 & Norman, Okla. & AGN & Homog. solid, pool & Neglig. & 1958 & \\
\hline Iowa State University & UTR-10 & Ames, Iowa & ARSS & Graphite/water & 10 & 1959 & \\
\hline Leland Stanford University & & Palo Alto, Calif. & GE & Pool & 10 & 1959 & \\
\hline University of Florida & UFTR & Gainesville, Fla. & GNEC & Graphite/water & 100 & 1959 & \\
\hline University of Wyoming & $\mathrm{L}-77$ & Laramie, Wyo. & AI & Homogeneous & Neglig. & 1959 & \\
\hline Virginia Polytechnıc Institute & UTR-10 & Blacksburg, Va. & ARSS & Graphite/water & 100 & 1959 & \\
\hline $\begin{array}{l}\text { West Virginia University, College of } \\
\text { Engineering }\end{array}$ & AGN-211-103 & Morgantown, W. Va. & AGN & Homog. solid, pool & Neglig. & 1959 & \\
\hline Worcester Polytechnic Institute & & Worcester, Mass. & GE & Pool & 1 & 1959 & \\
\hline North Carolina State University & & Raleigh, N. C. & Cook & Graphite/water & 10 & 1960 & \\
\hline $\begin{array}{l}\text { University of Calıfornia at Los Angeles, } \\
\text { College of Engineerıng }\end{array}$ & Educator & Los Angeles, Calif. & $A M F$ & Graphite/water & 100 & 1960 & \\
\hline Unıversity of Illinols & TRIGA-Mk II & $\begin{array}{l}\text { Urbana-Champaign, } \\
\text { Ill. }\end{array}$ & GDC & $\mathrm{U}-\mathrm{Zr}$ hydrıde, tank & 250 & 1960 & \\
\hline University of Maryland & UMNE - 1 & College Park, Md. & $\mathrm{AC}$ & Tank & 10 & 1960 & \\
\hline Unıversity of Virginia & & Charlottesville, Va. & Owner-B\&W & Pool & 1,000 & 1960 & \\
\hline University of Wisconsm & & Madison, Wis. & GE & Pool & 250 & 1960 & \\
\hline $\begin{array}{l}\text { Nuclear Science Center Reactor, Texas A\&M } \\
\text { University }\end{array}$ & NSCR & College Station, Tex. & Convalr & Pool & 100 & 1961 & \\
\hline Ohio State University & & Columbus, Ohıo & Lockheed & Pool & 10 & 1961 & \\
\hline University of Kansas & Model 4180 & Lawrence, Kans. & BAC & Pool & 10 & 1961 & \\
\hline University of Missouri at Rolla & & Rolla, Mo. & $\mathrm{CW}$ & Pool & 200 & 1961 & \\
\hline University of Washington & Educator & Seattle, Wash. & AMF & Graphite/water & 10 & 1961 & \\
\hline Washington State Unıversity & & Pullman, Wash. & GE & Pool & 100 & 1961 & \\
\hline
\end{tabular}


western New York Nuclear Kesearch

PULSTAR

Buffalo, N. Y.

Pool

$2,000 \quad 1961$ Cornell University

Kansas State University

TRIGA -Mk II Ithaca, N. Y.

Manhattan, Kans.

$\mathrm{U}-\mathrm{Zr}$ hydride

$U-Z r$ hydride, tank

Pool

West Lafayette, Ind. Lockheed

L-77

Reno, Nev.

Homogeneous

$U-Z r$ hydride, tank

Homog. solid

New York Caty

New York University ${ }^{38}$

AGN-201M-105

GDC

Georg1a Tech. Research Reactor

GTRR Atlanta, Ga

Heavy water

$100 \quad 1962$

PSTR

Unıversity Park, $\mathrm{Pa}$.

GNEC

State University)
State TRIGA

University of California ${ }^{32}$

University of Missour 1

TRIGA-Mk II

Berkeley, Calıf

Columbia, Mo.

BEING BUILT

Mississippi State Unıversity ${ }^{39}$

Columbia University

Lowell Technological Institute

Oregon State University

Brigham Young University

PLANNED

North Carolına State University

RRR

TRIGA-Mk II

TRIGA-Mk III

L -77

PULSTAR
State College, Miss.

New York City

Lowell, Mass.

Corvallis, Oreg.

Provo, Utah

Raleıgh, N. C
Tank

GDC

GDC

Owner-IC

Tank
Tank

Homogeneous

$\mathrm{U}-\mathrm{Zr}$ hydrıde, tank

Pool

Tank

Homogeneous

Pool

AM F

\section{DEFENSE POWER-REACTOR APPLICATIONS}

\section{PART II MILITARY REACTORS}

\section{A. Remote Installations}

\begin{tabular}{|c|c|c|c|c|c|c|c|c|}
\hline \multirow[b]{2}{*}{ Name (all owned by DOD) } & \multirow[b]{2}{*}{ Destgnation ${ }^{40}$} & \multirow[b]{2}{*}{ Location } & \multirow[b]{2}{*}{$\begin{array}{c}\text { Principal } \\
\text { nuclear contractor }\end{array}$} & \multirow[b]{2}{*}{ Tуре } & \multicolumn{2}{|c|}{ Power $^{1}$} & \multirow[b]{2}{*}{$\begin{array}{l}\text { Start- } \\
\quad \text { up }\end{array}$} & \multirow[b]{2}{*}{$\begin{array}{l}\text { Shut- } \\
\text { down }\end{array}$} \\
\hline & & & & & $\begin{array}{l}\text { Plant, } \\
\text { net kw(e) }\end{array}$ & $\begin{array}{l}\text { Reactor, } \\
\text { kw(t) }\end{array}$ & & \\
\hline \multicolumn{9}{|l|}{ SHUT DOWN OR DISMANTLED } \\
\hline Portable Medium Power Plant No. $2 \mathrm{~A}^{41}$ & PM-2A & $\begin{array}{l}\text { Camp Century, } \\
\text { Greenland }\end{array}$ & Alco & Pressurızed water & 1,560 & 10,000 & 1960 & 1963 \\
\hline \multicolumn{9}{|l|}{ OPERABLE } \\
\hline $\begin{array}{l}\text { Stationary Medium Power Plant No. } 1 \\
\text { (Formerly APPR-1) }\end{array}$ & SM-1 & Ft. Belvoir, Va. & Alco & Pressurized water & 1,750 & 10,000 & 1957 & \\
\hline Portable Medium Power Plant No. $1^{42}$ & PM-1 & Sundance, Wyo. & Martın & Pressurized water & 1,000 & 9,370 & 1962 & \\
\hline Portable Medium Power Plant No. 3A & PM-3A & $\begin{array}{l}\text { McMurdo Sound, } \\
\text { Antarctica }\end{array}$ & Martın & Pressurized water & 1,500 & 9,510 & 1962 & \\
\hline $\begin{array}{l}\text { Stationary Meduum Power Plant No. } 1 \mathrm{~A}^{43} \\
\text { BEING BUILT }\end{array}$ & SM-1A & Fort Greely, Alaska & Alco & Pressurized water & 1,640 & 20,000 & 1962 & \\
\hline $\begin{array}{l}\text { "Sturgis" Floating Nuclear Power } \\
\text { Plant }\end{array}$ & $\mathrm{MH}-1 \mathrm{~A}$ & Fort Belvolr, Va & Martın & Pressurızed water & 10,000 & 45,000 & 1967 & \\
\hline
\end{tabular}




\section{B. Propulsion (Naval)}

\begin{tabular}{|c|c|c|c|c|}
\hline Name (all owned by $U S \mathrm{Naz} y$ ) & Designation & Shpbualder & $\begin{array}{c}\text { Start } \\
u p \\
\end{array}$ & $\begin{array}{l}\text { Shut - } \\
\text { doun }\end{array}$ \\
\hline \multicolumn{5}{|l|}{ SHUT DOWN OR DISMANTLED } \\
\hline SEAWOLF Sodum Reactor ${ }^{45}$ & & Electric Boat (Groton) & 1956 & 1959 \\
\hline $\begin{array}{l}\text { USS THRESHER } \\
\text { OPERABLE }\end{array}$ & SSN593 & Portsmouth & 1961 & 1963 \\
\hline USS NAUTILUS & SSN571 & Electric Boat (Groton) & 1954 & \\
\hline USS SEAWOLF ${ }^{45}$ & SSN575 & Electric Boat (Groton) & 1960 & \\
\hline USS SKATE & SSN578 & Electric Boat (Groton) & 1957 & \\
\hline USS SWORDFISH & SSN579 & Portsmouth & 1958 & \\
\hline USS SARGO & SSN583 & Mare Island & 1958 & \\
\hline USS SEADRAGON & SSN584 & Portsmouth & 1959 & \\
\hline USS SKIPJACK & SSN585 & Electric Boat (Groton) & 1958 & \\
\hline USS TRITON (2 reactors) & SSN586 & Electric Boat (Groton) & 1959 & \\
\hline USS HALIBUT & SSN587 & Mare 1sland & 1959 & \\
\hline USS SCAMP & SSN588 & Mare Island & 1961 & \\
\hline USS SCORPION & $\operatorname{SSN} 589$ & Electric Boat (Groton) & 1960 & \\
\hline USS SCULPIN & SSN590 & Ingalls & 1961 & \\
\hline USS SHARK & SSN591 & Newport News & 1960 & \\
\hline USS SNOOK & SSN592 & Ingalls & 1961 & \\
\hline USS PERMTT & SSN594 & Mare Island & 1962 & \\
\hline USS PLUNGER & SSN 595 & Mare Island & 1962 & \\
\hline USS BARB & $\operatorname{SSN} 596$ & Ingalls & 1963 & \\
\hline USS TULLIBEE & SSN597 & Electric Boat (Groton) & 1960 & \\
\hline USS GEORGE WASHINGTON & SSBN598 & Electric Boat (Groton) & 1959 & \\
\hline USS PATRICK HENRY & SSBN599 & Electric Boat (Groton) & 1960 & \\
\hline USS THEODORE ROOSEVELT & SSBN600 & Mare Island & 1960 & \\
\hline USS ROBERT E LEE & SSBN601 & Newport News & 1960 & \\
\hline USS ABRAHAM LINCOLN & SSBN602 & Portsmouth & 1960 & \\
\hline USS POLLACK & SSN603 & NYSC & 1963 & \\
\hline USS HADDO & SSN604 & NYSC & 1964 & \\
\hline USS TINOSA & SSN606 & Portsmouth & 1963 & \\
\hline USS DACE & SSN607 & Ingalls & 1963 & \\
\hline USS ETHAN ALLEN & SSBN608 & Electric Boat (Groton) & 1961 & \\
\hline USS SAM HOUSTON & SSBN609 & Newport News & 1961 & \\
\hline USS THOMAS A EDISON & SSBN610 & Electric Boat (Groton) & 1961 & \\
\hline USS JOHN MARSHALL & SSBN611 & Newport News & 1962 & \\
\hline USS GUARDFISH & SSN612 & NYSC & 1966 & \\
\hline USS FLASHER & SSN613 & Electric Boat (Groton) & 1966 & \\
\hline USS LAFAYETTE & SSBN616 & Electric Boat (Groton) & 1963 & \\
\hline USS ALEXANDER HAMLTON & SSBN617 & Electric Boat (Groton) & 1963 & \\
\hline USS THOMAS JEFFERSON & SSBN618 & Newport News & 1962 & \\
\hline USS ANDREW JACKSON & SSBN619 & Mare Island & 1963 & \\
\hline USS JOHN ADAMS & SSBN620 & Portsmouth & 1964 & \\
\hline USS JAMES MONROE & SSBN622 & Newport News & 1963 & \\
\hline USS NATHAN HALE & SSBN623 & Electric Boat (Groton) & 1963 & \\
\hline USS WOODROW WILSON & SSBN624 & Mare Island & 1963 & \\
\hline USS HENRY CLAY & SSBN625 & Newport News & 1963 & \\
\hline USS DANIEL WEBSTER & SSBN626 & Electric Boat (Groton) & 1964 & \\
\hline USS JAMES MADISON & SSBN627 & Newport News & 1964 & \\
\hline USS TECUMSEH & SSBN628 & Electric Boat (Groton) & 1964 & \\
\hline USS DANIEL BOONE & SSBN629 & Mare Island & 1963 & \\
\hline USS JOHN CALHOUN & SSBN630 & Newport News & 1964 & \\
\hline USS ULYSSES S GRANT & SSBN631 & Electric Boat (Groton) & 1964 & \\
\hline USS VON STEUBEN & SSBN632 & Newport News & 1964 & \\
\hline USS CASIMIR PULASKI & SSBN633 & Electric Boat (Groton) & 1964 & \\
\hline USS STONEWALL JACKSON & SSBN634 & Mare Island & 1964 & \\
\hline USS SAM RAYBURN & $\operatorname{SSBN} 635$ & Newport News & 1964 & \\
\hline USS NATHANAEL GREENE & SSBN636 & Portsmouth & 1964 & \\
\hline USS STURGEON & SSN637 & Electric Boat (Groton) & 1966 & \\
\hline USS BENIAMIN FRANKLIN & SSBN640 & Electric Boat (Groton) & 1965 & \\
\hline
\end{tabular}

\begin{tabular}{|c|c|c|c|c|}
\hline Name (all ouncd by $U S \mathrm{Na} y$ ) & Designation & Shopbulder & $\begin{array}{c}\text { Start } \\
\text { up }\end{array}$ & $\begin{array}{l}\text { Shut - } \\
\text { down }\end{array}$ \\
\hline USS SIMON BOLIVAR & S১BN641 & Newport News & 1965 & \\
\hline USS KAMEHAMEHA & SSBN642 & Mare Island & 1965 & \\
\hline USS GEORGE BANCROFT & SSBN643 & Electric Boat (Groton) & 1965 & \\
\hline USS LEWIS AND CLARK & SSBN644 & Newport News & 1965 & \\
\hline USS JAMES K POLK & SSBN645 & Electric Boat (Groton) & 1966 & \\
\hline USS QUE ENFISH & SSN651 & Newport News & 1966 & \\
\hline USS GEORGE C MARSHALL & SSBN654 & Newport News & 1966 & \\
\hline USS HENRY L STIMSON & SSBN655 & Electric Boat (Groton) & 1966 & \\
\hline $\begin{array}{l}\text { USS GEORGE WASHINGTON } \\
\text { CARVER }\end{array}$ & SSBN656 & Newport News & 1966 & \\
\hline USS LONG BEACH (2 reactors) & CGN9 & Bethlehem & 1961 & \\
\hline USS ENTERPRISE ( 8 reactors) & CVAN65 & Newport News & 1960 & \\
\hline USS BAINBRIDGE (2 reactors) & DLGN25 & Bethlehem & 1962 & \\
\hline USS FRANCIS SCOTT KEY & SSBN657 & Electric Boat (Groton) & 1966 & \\
\hline USS MARIANO G VALLEJO & SSBN658 & Mare Island & 1966 & \\
\hline \multicolumn{5}{|l|}{ BEING BUILT } \\
\hline JACK & SSN605 & Portsmouth & & \\
\hline GREENLING & SSN614 & GD (Quincy) & & \\
\hline GATO & SSN615 & GD (Quincy) & & \\
\hline HADDOCK & SSN621 & Ingalls & & \\
\hline WHALE & SSN638 & Electric Boat (Quncy) & & \\
\hline TAUTOG & SSN639 & Ingalls & & \\
\hline GRA YLING & SSN646 & Portsmouth & & \\
\hline POGY & SSN647 & NYSC & & \\
\hline ASPRO & SSN648 & Ingalls & & \\
\hline SUNFISH & $\operatorname{SSN} 649$ & GD (Quincy) & & \\
\hline PARGO & SSN650 & Electric Boat (Groton) & & \\
\hline PUFFER & SSN652 & Ingalls & & \\
\hline RAY & SSN653 & Newport News & & \\
\hline WILL ROGERS & SSBN659 & Electric Boat (Groton) & & \\
\hline SAND LANCE & $\operatorname{SSN} 660$ & Portsmouth & & \\
\hline LAPON & $\operatorname{SSN} 661$ & Newport News & & \\
\hline GURNARD & SSN662 & Mare Island & & \\
\hline HAMMERHEAD & SSN663 & Newport News & & \\
\hline SEA DEVIL & $\operatorname{SSN} 664$ & Newport News & & \\
\hline GUITARRO & $\operatorname{SSN} 665$ & Mare Island & & \\
\hline HAWKBILL & SSN666 & Mare Island & & \\
\hline BERGALL & $\operatorname{sSN} 667$ & Electruc Boat (Groton) & & \\
\hline SPADF FISH & $\operatorname{sSN} 668$ & Newport News & & \\
\hline SEAHORSE & SSN669 & Electric Boat (Groton) & & \\
\hline Submarine & SSN670 & Newport News & & \\
\hline NARWHAL & SSN671 & Electric Boat (Groton) & & \\
\hline PIN TADO & SSN672 & Mare Island & & \\
\hline Submarine & SSN673 & Electric Boat (Groton) & & \\
\hline Submas ine & SSN674 & Electric Boat (Groton) & & \\
\hline Subman ine & SSN675 & Electrie Boat (Groton) & & \\
\hline Subnarıne & SSN676 & Electric Boat (Groton) & & \\
\hline TRUXTUV (2 reactors) & DLGN35 & VYSC & & \\
\hline $\begin{array}{l}\text { Deep Submersence Research } \\
\text { Vehucle }\end{array}$ & NR 1 & Electric Boat (Groton) & & \\
\hline Aircraft Carrier & CVAN68 & Unassigned & & \\
\hline \multicolumn{5}{|l|}{ PLA UNED } \\
\hline Guided Missile Figate ${ }^{47}$ & DLGN & Unassigned & & \\
\hline Submarine & $\operatorname{SS\wedge } 677$ & Unassigned & & \\
\hline Submar ıne & SS^678 & Unassigned & & \\
\hline Submarıne & SSN679 & Unassigned & & \\
\hline Submarıne & SSN680 & Unassigned & & \\
\hline Submarine & SSN681 & Unassigned & & \\
\hline Submarine & SSA682 & Unassigned & & \\
\hline
\end{tabular}




\section{DEVELOPMENTAL POWER}

\section{A. Electric-Power Experiments and Prototypes for Remote Installations}

\begin{tabular}{|c|c|c|c|c|c|c|c|c|}
\hline \multirow[b]{2}{*}{$\begin{array}{c}\text { Name (all ound bu } A \Gamma C \\
\text { creeptas noted ) }\end{array}$} & \multirow[b]{2}{*}{ Destgnation 40} & \multirow[b]{2}{*}{ Location } & \multirow[b]{2}{*}{$\begin{array}{c}\text { Principal } \\
\text { nuclear contractor }\end{array}$} & \multirow[b]{2}{*}{$T, p e$} & \multicolumn{2}{|c|}{ Poner } & \multirow[b]{2}{*}{$\begin{array}{l}\text { Start- } \\
\text { up }\end{array}$} & \multirow[b]{2}{*}{$\begin{array}{l}\text { Shut- } \\
\text { doun }\end{array}$} \\
\hline & & & & & $\begin{array}{c}\text { Plant, } \\
\text { net } k u(c)\end{array}$ & $\begin{array}{c}\text { Rcactor, } \\
k u(t)\end{array}$ & & \\
\hline \multicolumn{9}{|l|}{ SHUT DOWN OR DISMANTLED } \\
\hline Stationary Low Power Plant No. 1 & SL-1 & NRTS, Idaho & ANL & Bosling water & 300 & 2,200 & 1958 & 1961 \\
\hline Gas Cooled Reactor Experıment ${ }^{48}$ & GCRE & NRTS, Idaho & AGN & $\begin{array}{l}\text { Gas cooled, light } \\
\text { water moderated }\end{array}$ & No elec. & 2,200 & 1960 & 1962 \\
\hline Mobile Low Power Plant No. $1^{49}$ & $M L-1$ & NRTS, Idaho & AGN & $\begin{array}{l}\text { Gas cooled, light } \\
\text { water moderated }\end{array}$ & 300 & 3,300 & 1961 & 1965 \\
\hline
\end{tabular}

\section{B. Propulsion Experiments and Prototypes}

\begin{tabular}{|c|c|c|c|c|c|c|c|}
\hline Name (all ouned bi $A H($ except as noted) & Desagnation & Location & $\begin{array}{l}\text { Principal } \\
\text { nuclcar } \\
\text { contractor }\end{array}$ & Tupe & $\begin{array}{c}\text { Power, } \\
k w(t)\end{array}$ & $\begin{array}{c}\text { Start - } \\
u p\end{array}$ & $\begin{array}{l}\text { Shut - } \\
\text { down }\end{array}$ \\
\hline
\end{tabular}

\section{B.1 Naval}

SHUT DOWN OR DISMANTLED

Submarine Intermediate Reactor Mark

\section{S1W}

A1W

AIG

S1C

Natural Circulation Test Plant

S5G

West Mllton, N. Y.

GE

Sodium

1955

NRTS, Idaho

West Muton, $N$ Y

Windsor, Conn.

West Multon, N. Y.

NRTS, Idaho

ARE

HTRE-1

HTRE - 2

HTRE -3

Oak Ridge, Tenn.

NRTS, Idaho

NRTS, Idaho

Heat Transfer Reactor Experıment No. 2

NRTS, Idaho

West.
West.
GE
Comb.
GE
GE

Pressurized water

Pressurized water

Pressurized water

Pressurized water

Pressurized water

Pressurized water

\section{B.3 Missile (Project PLUTO)}

SHUT DOWN OR DISMANTLED

Experimental Propulsion Test Reactor

Experimental Propulsion Test Re $\ll$ tor $^{50}$

\section{TEST AND RESEARCH}

\section{A. Test}

SHUT DOWN OR DISMANT LED

Tower Shrelding Reactor No.

Nuclear Effects Reactor (AEC) 


\section{A. Test (Continued)}

\begin{tabular}{|c|c|c|c|c|c|c|c|}
\hline Name (all owned by $A E C$ except as noted) & Designatron & Locatron & $\begin{array}{c}\text { Principal } \\
\text { nuclear } \\
\text { contractor }\end{array}$ & Type & $\begin{array}{c}\text { Power, }{ }^{1} \\
k w(t)\end{array}$ & $\begin{array}{l}\text { Start- } \\
\text { up }\end{array}$ & $\begin{array}{l}\text { Shut- } \\
\text { down }\end{array}$ \\
\hline \multicolumn{8}{|l|}{ OPERABLE } \\
\hline Ground Test Reactor (USAF) & GTR & Ft. Worth, Tex. & Convair & Pool & 10,000 & 1953 & \\
\hline Aerospace Systems Test Reactor (USAF) & ASTR & Ft. Worth, Tex. & Convair & Light water & 10,000 & 1954 & \\
\hline Tower Shieldıng Reactor No. 2 & TSR-2 & Oak Rıdge, Tenn. & ORNL & Light water & 100 & 1960 & \\
\hline Nuclear Engıneerıng Test Reactor (USAF) & NETR & Dayton, Ohio & Maxon-AC & Tank & 10,000 & 1965 & \\
\hline \multicolumn{8}{|l|}{ B. Research } \\
\hline \multicolumn{8}{|l|}{ OPERABLE } \\
\hline Thermal Test Reactor No. 1 & TTR-1 & Schenectady, N. Y. & KAPL & Graphite & 10 & 1951 & \\
\hline Naval Research Reactor (USN) & NRR & Washington, D. C. & NRL & Pool & 1,000 & 1956 & \\
\hline U. S. Naval Post Graduate School (USN) & $A G N-201-100$ & Monterey, Calıf. & AGN & Homog. solid & Neglig. & 1956 & \\
\hline $\begin{array}{l}\text { Army Materials Research Reactor } \\
\quad(\text { Army Materials Research Agency, USA) }\end{array}$ & AMRR & Watertown, Mass. & $\mathrm{BAC}$ & Pool & 2,000 & 1960 & \\
\hline $\begin{array}{l}\text { Diamond Ordnance Radiation Facility } \\
\text { (Harry Diamond Laboratories, USA) }\end{array}$ & DORF & Sllver Spring, Md. & GDC & $U-Z r$ hydrıde & 100 & 1961 & \\
\hline $\begin{array}{l}\text { Armed Forces Radiobiology Research } \\
\text { Institute (DASA, DOD) }\end{array}$ & AFRRI & Bethesda, Md. & GDC & $\mathbf{U}-\mathrm{Zr}$ hydride, tank & 100 & 1962 & \\
\hline $\begin{array}{l}\text { Walter Reed Research Reactor (Walter Reed } \\
\text { Army Institute of Research, USA) }\end{array}$ & WRRR & Washington, D. C. & AI & Homogeneous & 50 & 1962 & \\
\hline $\begin{array}{l}\text { Fast Burst Reactor Facility (Army Missile } \\
\text { Test and Evaluation Directorate, USA) }\end{array}$ & FBRF & White Sands, N. Mex. & Kaman & Bare, fast & 10 & 1964 & \\
\hline Nuclear Effects Reactor (AEC) & Super KUKLA & NTS, Nev. & UCLRL & Prompt burst & Transient & 1964 & \\
\hline $\begin{array}{l}\text { Aberdeen Pulsed Reactor Facility (Ballistic } \\
\text { Research Laboratories, USA) }\end{array}$ & APRF & Aberdeen, Md. & UNC & Bare, fast & 10 & 1966 & \\
\hline
\end{tabular}

1. MATERIALS PRODUCTION

\section{PART III PRODUCTION REACTORS}

\begin{tabular}{|c|c|c|c|c|c|}
\hline Designation & Nuclear designer & Type & Location & Startup & Shutdown \\
\hline \multicolumn{6}{|l|}{ SHUT DOWN } \\
\hline R Reactor & du Pont & Heavy water & Savannah River Plant, Alken, S. C. & 1953 & 1964 \\
\hline H Reactor & $\mathrm{GE}$ & Graphite & Richland, Wash. & 1949 & 1965 \\
\hline F Reactor & du Pont & Graphite & Richland, Wash. & 1945 & 1965 \\
\hline \multicolumn{6}{|l|}{ OPERABLE } \\
\hline B Reactor & du Pont & Graphite & Richland, Wash. & 1944 & \\
\hline KW Reactor & GE & Graphite & Ruchland, Wash. & 1955 & \\
\hline $\begin{array}{l}\text { N Reactor }{ }^{51} \\
\text { P Reactor }\end{array}$ & $\begin{array}{l}\text { GE } \\
\text { du Pont }\end{array}$ & $\begin{array}{l}\text { Graphite } \\
\text { Heavy water }\end{array}$ & $\begin{array}{l}\text { Richland, Wash. } \\
\text { Savannah River Plant, Aiken, S. C. }\end{array}$ & $\begin{array}{l}1963 \\
1954\end{array}$ & \\
\hline K Reactor & du Pont & Heavy water & Savannah River Plant, Aiken, S. C. & 1954 & \\
\hline L Reactor & du Pont & Heavy water & Savannah River Plant, Alken, S. C. & 1954 & \\
\hline C Reactor & du Pont & Heavy water & Savannah River Plant, Alken, S. C. & 1955 & \\
\hline
\end{tabular}




\section{TEST}

OPERABLE

Hanford 305 Test Reactor

Savannah River Test P1le 305

\section{HTR}

Richland, Wash.

\section{POWER REACTORS}

\section{A. Central-Station Electric Power}

\section{PART IV REACTORS FOR EXPORT} Location nuclear contractor

Type

\begin{tabular}{ccc} 
Power $^{\prime}$ & \\
\hline Plant, & Reactor, & Start- \\
net kw(e) & $k w(t)$ & $u p$ \\
\hline
\end{tabular}

OPERABLE

Germany, Kahl Nuclear Power Statıon (Rhıne-Westphalıa Power Co., RWE) Italy, Garıghiano Nuclear Power Station (Project ENSI of SENN)

Japan, Government of, Japan Power Demonstration Reactor (JAERI) Italy (Project Enrico Fermi of SELNI, Edisonvolta)

France (Franco-Belgian Society for Nuclear Energy of Ardennes - SENA) West Germany (Kernkraftwerk-RWE-Bayernwerk or KRB)

Kahl-am-Main Punta Fiume (on Garıgliano River) Toka1-Mura Trıno Vercellese Givet (near Chooz) Gundremmingen (near Gunzburg)

BEING BUILT

Ind1a, Government of (Tarapur Nuclear Power Statıon)

Japan (Japan Atomic Power Company -- JAPCO No 2)

Spain, Zorita Nuclear Power Plant Unit No. 1

Spain, Government of (Nuclenor)

Switzerland, NOK Nuclear Electric Generatıng Statıon Unıt No. 1 PLANNED

Japan, Mihama Nuclear Power Statıon Unıt No. 1 (Kansal Electrıc Power Co )

Japan, Tokyo Electric Power Co Unit No.

Switzerland (Bernische Kraftwerk A.G -BKW)

\section{B. Propulsion}

\begin{tabular}{|c|c|c|c|c|c|}
\hline Name & Owner & Designer & Designation & Type & $\begin{array}{c}\text { Start- } \\
u p\end{array}$ \\
\hline \multicolumn{6}{|l|}{ OPERABLE } \\
\hline S5W for HMS Dreadnought & Great Britain & West. & S5W & Pressurized water & 1962 \\
\hline
\end{tabular}


2. TEST, RESEARCH, AND TEACHING

A. General Irradiation Test

\begin{tabular}{|c|c|c|c|c|c|}
\hline Owner & Location & $\begin{array}{c}\text { Principal } \\
\text { nuclear contractor }\end{array}$ & Type & $\begin{array}{c}\text { Power, }{ }^{1} \\
k w(t)\end{array}$ & $\begin{array}{l}\text { Start- } \\
\text { up }\end{array}$ \\
\hline \multicolumn{6}{|l|}{ OPERABLE } \\
\hline Japan, Government of (Atomic Energy Research Institute) & Toka1-Mura & AMF & Heavy water, tank & 10,000 & 1960 \\
\hline Netherlands, Government of (Reactor Center) & Petten & $\mathrm{AC}$ & Tank (MTR) & 30,000 & 1961 \\
\hline South Africa, Government of (Atomic Energy Board) & $\begin{array}{l}\text { Pelindaba (near } \\
\text { Pretoria) }\end{array}$ & $\mathrm{AC}$ & Tank & 20,000 & 1965 \\
\hline Sweden, Government of (Atomic Energy Company) & Studsvik & $\mathrm{AC}$ & Tank (MTR) & 30,000 & 1961 \\
\hline
\end{tabular}

\section{B. General Research}

\section{OPERABLE}

Australıa (Atomic Energy Commission)

Austria, Government of (Se1bersdorf Research Center)

Colombia (Colombian Institute of Nuclear Affairs)

Denmark, Government of (Atomic Energy Commission)

Denmark, Government of (Atomic Energy Commission)

Germany, Federal Republic of (Society for the Utilization of Nuclear

Energy in Shipbuilding and Navigation, Inc.)

Germany (Interatom, Inc.) (USAEC Middle East Exhıbit Program) ${ }^{54}$

Greece, Government of (Atomic Energy Commission)

Indones ia (Institute for Atomic Energy)

Israel, Government of (Atomic Energy Commission)

Italy (C.A.M.E.N.)

Italy, Government of (National Committee for Nuclear Energy)

Italy, Government of (National Committee for Nuclear Energy)

Italy (SORIN Nuclear Center)

Japan, Government of (Atomic Energy Research Institute)

Korea, Government of (Atomic Energy Commission)

Pakistan, Government of (Atomic Energy Commission)

Philippines, Government of (National Science Development Board)

Portugal, Government of (Nuclear Energy Board)

Spain, Government of (Nuclear Energy Board)

Switzerland, Government of (Reactor, Inc $)^{55}$

Turkey, Government of (Atomic Energy Commission)

Venezuela, Government of (Institute of Neurology and Brain Research) ${ }^{56}$

Vietnam, Government of (Institute of Nuclear Research)

West Berlın, City of (Institute for Nuclear Research)

Yugoslavia, Federal People's Government of (Josef Stefan Nuclear Institute)

Lucas-Heights,

New South Wales

Selbersdorf

Bogotá

Riso

Riso

Geesthacht

Dussburg

Athens

Bandung

Nahal Soreq

Leghorn

Ispra

Rome

Saluggia

Toka1-Mura

Seoul

Islamabad

Quezon City

Sacavém

Moncloa

Wuerenlingen

Istanbul

Caracas

Dalat

West Berlin

Ljubljana

ARSS

UTR-10

10

1961

AMF

Lockheed

AI

FW

B\&W

Pool

Pool

L-55

Tank

Pool

5,000

10

5,000

5,000

L-77

AMF

GDC

GDC

AMF

$B \& W$
$A C$

GDC

AMF

AI
GDC

AMF

GE

AMF

GE

ORNL

AMF

GE

GDC

AI

BEING BUILT

Italy (CNEN)

Mexico (National Instıtute for Nuclear Energy)

TRIGA-Mk II

Neglig.

Pool

Pool

, tank $\quad 5,000$

Pool

L-54

TRIGA-Mk II

Pool

Pool

Pool

Pool

Pool

Pool

Pool

TRIGA-Mk II

L-54

TRIGA-Mk II

1961

1964

1959

100

1,000

50

$100 \quad 1962$

$5,000 \quad 1965$

$1,000 \quad 1963$

$1,000 \quad 1961$

3,000

$3,000 \quad 1958$

$\begin{array}{ll}1,000 & 1955 \\ 1,000 & 1962\end{array}$

$\begin{array}{ll}1,000 & 1962 \\ 3,000 & 1960\end{array}$

$250 \quad 1963$

250

$50 \quad 1958$

1966 


\begin{tabular}{|c|c|c|c|c|c|}
\hline Ouner & Location & $\begin{array}{l}\text { Principal } \\
\text { nuclear contractor }\end{array}$ & Type & $\begin{array}{l}\text { Power, } \\
\text { kw }(t)\end{array}$ & $\begin{array}{l}\text { Start- } \\
u p\end{array}$ \\
\hline \multicolumn{6}{|l|}{ OPERABLE } \\
\hline Austria, Government of (Vienna Polytechnic Institute) & Vienna & GDC & TRIGA-Mk II & 100 & 1962 \\
\hline Brazıl, Government of (Unuversity of São Paulo) & São Paulo & $B \& W$ & Pool & 5,000 & 1957 \\
\hline Brazil (University of Minas Gerais) & Belo Horizonte & GDC & TRIGA-Mk I & 30 & 1960 \\
\hline Canada (McMaster Unıversity) & Hamilton, Ont. & AMF & Pool & 1,000 & 1959 \\
\hline China, Republic of (National Tsing-Hua University) & Hsinchu & GE & Pool & 1,000 & 1961 \\
\hline Congo, Government of Kinshasa (University of Louvanıum) ${ }^{57}$ & Kinshasa & GDC & TRIGA-Mk I & 50 & 1959 \\
\hline Finland, Government of (Institute of Technology) & Helsinkı & GDC & TRIGA-Mk II & 100 & 1962 \\
\hline Germany, Federal Republic of (Technical University of Munich) & Munich & AMF & Pool & 1,000 & 1957 \\
\hline $\begin{array}{l}\text { Germany, Federal Republic of (Universities of Frankfurt and } \\
\text { Darmstadt) }\end{array}$ & Frankfurt & AI & $L-54$ & 50 & 1958 \\
\hline Germany (Johannes Gutenberg, University of Mainz) & Mainz & GDC & TRIGA-Mk II & 30 & 1965 \\
\hline Italy (University of Milan) & Milan & AI & L-54 & 50 & 1959 \\
\hline Italy (Unuversity of Palermo) & Palermo & AGN & $201-110$ & Neglig. & 1960 \\
\hline Italy (University of Pavia) & Pavia & GDC & TRIGA - Mk II & 250 & 1966 \\
\hline Japan (Kyoto Unıversity) & Kyoto & IC & Tank & 1,000 & 1964 \\
\hline Japan (Kinkı Unıversity) & Osaka & ARSS & UTR-10 & Neglig. & 1961 \\
\hline Japan (Musashı Unıversity) & Kawasakı City & GDC & TRIGA-Mk II & 100 & 1963 \\
\hline Japan (Rıkkyo University) & Yokosuka City & GDC & TRIGA-Mk $\amalg$ & 100 & 1961 \\
\hline Nether lands, Government of (Delft Technical University) ${ }^{58}$ & Delft & $\mathrm{AMF}$ & Pool & 100 & 1963 \\
\hline Switzerland (University of Basel) ${ }^{59}$ & Basel & AGN & $211-100$ & Neglig. & 1958 \\
\hline Switzerland (University of Geneva) ${ }^{60}$ & Geneva & AGN & $201-111$ & Neglig. & 1958 \\
\hline Thailand (Chulalongkorn University) & Bangkok & $\mathrm{CW}$ & Pool & 1,000 & 1962 \\
\hline United Kıngdom (Queen Mary College, London University) & London & ARSS & UTR-B & Neglig. & 1964 \\
\hline United Kıngdom, Government of (Scottısh Research Reactor Center) & East Kilbirde & ARSS & UTR-100 & 100 & 1963 \\
\hline $\begin{array}{l}\text { Uruguay (Unıversity of Montevideo) }{ }^{61} \\
\text { BEING BUILT }\end{array}$ & Montevideo & Lockheed & Pool & 10 & 1960 \\
\hline \multicolumn{6}{|l|}{ BEING BUILT } \\
\hline Iran, Government of (University of Tehran) & Tehran & AMF & Pool & 5,000 & 1967 \\
\hline
\end{tabular}

\section{IDENTIFICATION OF FACILITIES}

\section{PART V CRITICAL ASSEMBLY FACILITIES}

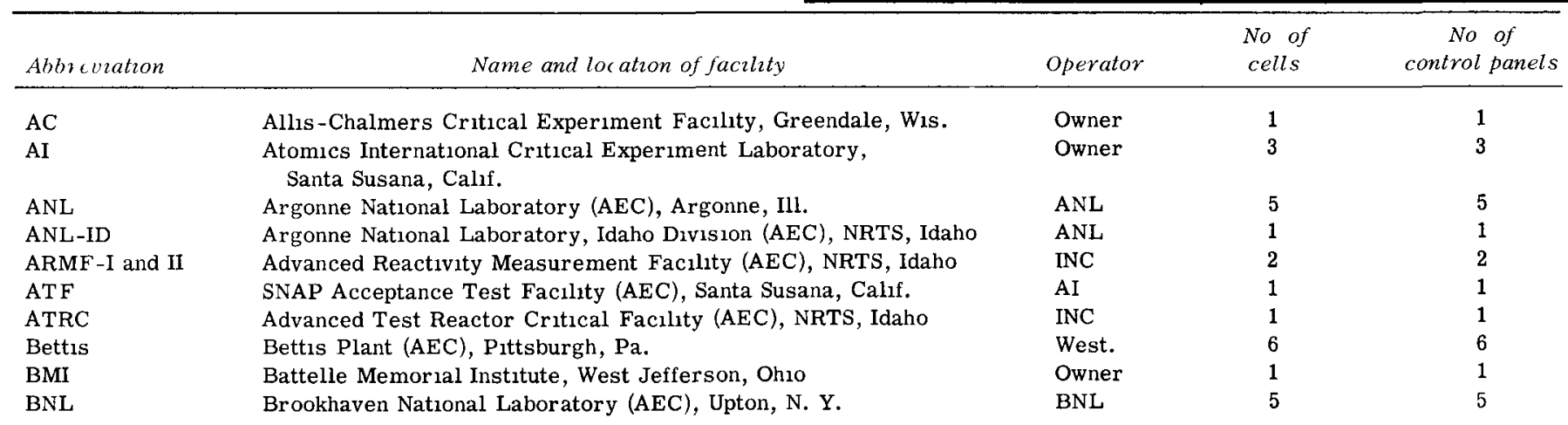




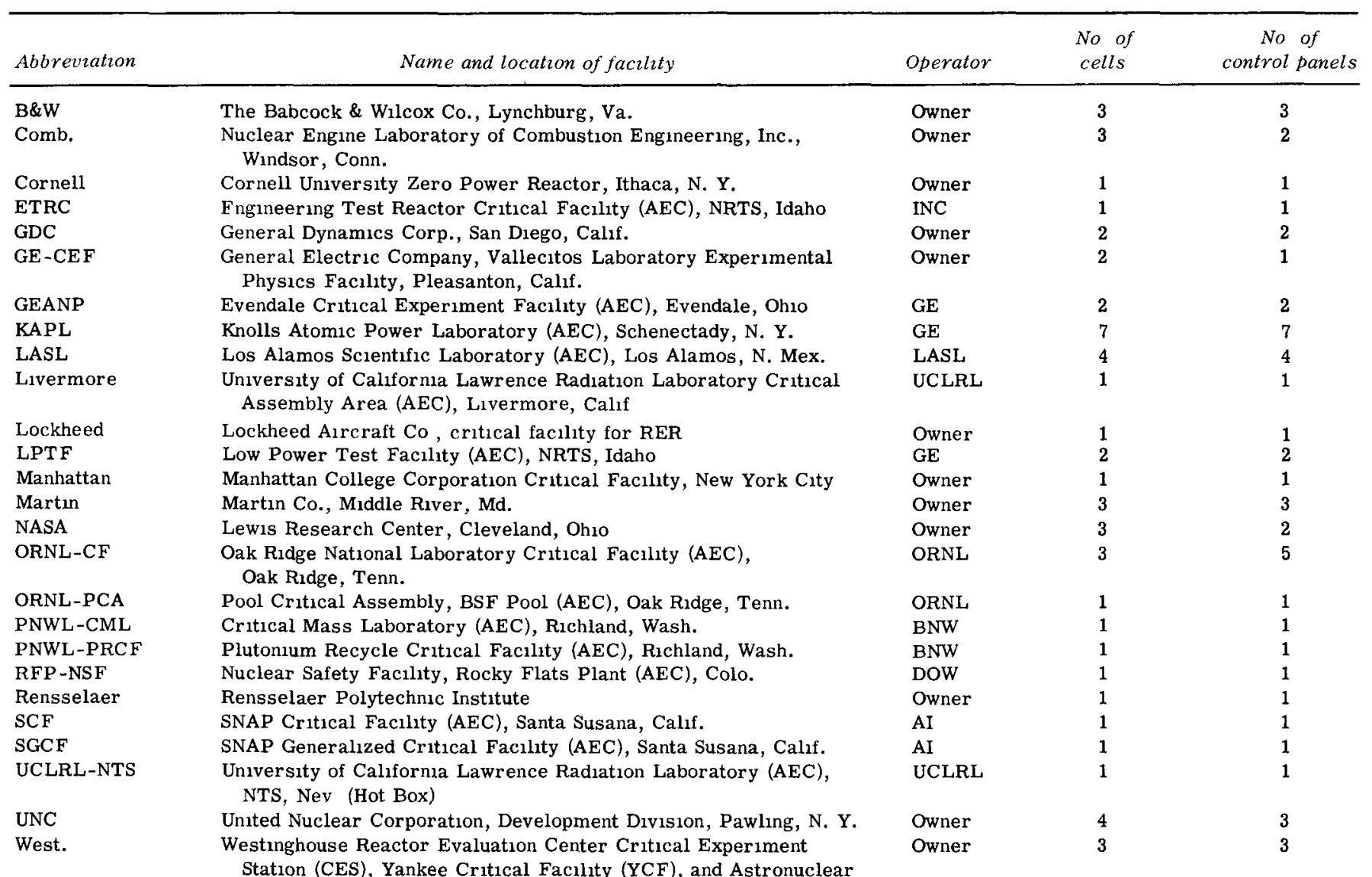

\section{IDENTIFICATION OF EXPERIMENTS AND STUDIES}

\section{A. Civilian}

\begin{tabular}{llr}
\hline Facility & Subject of current experiment or study & Designation \\
\hline OPERABLE & & Startup \\
\hline AC & Vacant & CRBR-CX \\
AI, AETR & Nuclear properties of epithermal, fast, and coupled & AETR \\
AI SCFA & reactor systems & 1959 \\
Nuclear oroperties of sodium - graphite svstems & SCGA
\end{tabular}


ANL, Bullding 316 , Cell $B^{62}$
ANL, Buxlding 316 , Cell $C^{62}$

ANL-ID

ARMF, ETR-MTR Area (INC)

ATRC, ATR Area (INC)

Bett1s

Bettis

BMI, Cell 1

BNL, Bulding T-526

BNL, Bulding T -526A

B\&W, Cell 1

$B \& W$, Cell $2^{63}$

B\&W, Cell $3^{63}$

Comb., Bullding 1, Cell 1

Comb., Bullding 2, Cell 1

Comb., Bullding 2, Cell 2

Cornell

ETRC, ETR-MTR Area (INC)

GDC

GDC

GE, CEF

LASL, Kuva I

LASL, Kıva III

LASL, Pit Bldg , TA-35

LPTF, Cell 1

LPTF, Cell 2

Manhattan

Martın, Cell 1

Martın, Cell 2

NASA, Materjals and

Stresses Bullding 64

NASA - ZPR-II ${ }^{64}$

ORNL-CF, Bulddng 9213, Cell E

ORNL-CF, Building 9213, Cell $\mathrm{S}$

ORNL-CF, Bullding 9213, Cell W

ORNL-PCA, Bullding 3010

PNWL-CML

PNWL-PRCF

SCF

SGCF

Renssalaer
Contains ZPR-7, high-conversion $\left(\mathrm{H}_{1}-\mathrm{C}\right)$ critical experiments

Flux-trap configuration critical studies for AARR

Fineriments

Reactor-physics constants and reactivity changes caused by test-reactor irradiation

ATR physics, core-loading and core-design measurements Critical experiments

LWB physics

Experıments with plastic-moderated assembly

Exponential and critical assembly

Exponential and critical assembly

Advanced Test Reactor criticals

Spectral Shuft Control Reactor

Small-lattice experiment

Vacant

Vacant

Vacant

Reactor-physics research

ETR physics, core loading and core design

Neutronic feasibility studies of enriched tungsten watermoderated reactors for nuclear rockets

Neutron yield, pulse shape, and spectra measurements, small electronic component irradiation

Thermal and mixed-spectrum critical assembly

Cold criticals for ROVER reactors

Cold critical operation of ROVER test reactors, environmental chamber, and general-purpose critical assembly

Criticals for UHTREX and Molten Plutonium reactors

Fast-spectrum refractory metals experıment

ARMF-I

and II

ATRC

CCFA

LWBCC

BMI-CX

ZEPR

ETRC

1957

APFA -

1965

TCA-MSCA

1961

ZEPO and Honeycomb

1957

1962

1965

710

1963

Critical experiment assembly

Martın power-reactor experiments

Homogeneous dispersions in a plastic of uranium, stanless steel, and boron

NASA Test Reactor criticai experıments (NASA-ZPR-I system)

NASA Zero Power Reactor II solution type critical

Uranum - paraff $1 n$ experiments related to physics and safety of homogeneous reactors

Comparison of nuclear properties of $U^{235}$ and $U^{233}$

Reactor-physics and nuclear-safety studies with homogeneous uranium solutions, slightly enriched-uranium lattices in water, TSR-2 tests; investigations of neutron absorbers as poisons in chemical-processing equipment Physics research on reactivity effects

Plutonium criticals

1961

Plutonium recycle criticals

Criticals for SNAP reactors

Critical facility for flight-system acceptance testing

1963

1963

Critical experiment assembly 
2. IDENTIFICATION OF EXPERIMENTS AND STUDIES

A. Civilian (Continued)

\begin{tabular}{cllr}
\hline Facility & Subject of current experiment or study & Designation & Startup \\
\hline & & PLATR & 1959 \\
UNC, Cell 1 & Pawling lattice test rig & & 1958 \\
West., CES & Reactor-fuel-measurement facility design & 1964 \\
West., ANEF & NERVA reactor experiments & NRX-CX & 1957 \\
West., YCF & Loose-lattice critical experiments & HTGRE & 1966 \\
GDC & High temperature gas cooled reactor experiment & & \\
BEING BUILT & PPPR & 1968 \\
ANL-ID & Plutonium criticals for fast reactors & APFA-III & 1967
\end{tabular}

B. Military

\begin{tabular}{|c|c|c|c|c|}
\hline Faciluty & Subject of current experiment or study & Designation & Startup & Shutdown \\
\hline \multicolumn{5}{|l|}{ OPERABLE } \\
\hline Bettıs & Surface-ship physics ${ }^{65}$ & SS-CF & 1957 & \\
\hline Bettis & High-temperature physics and mock-up & HTTF & 1959 & \\
\hline Bettis & Physics measurements & CCFB & 1960 & \\
\hline Bettis & Clean critıcal experıments ${ }^{65}$ & $\mathrm{CCX}$ & 1961 & \\
\hline GEANP - 1 & Vacant & & & \\
\hline GEANP - 2 & Vacant & & & \\
\hline KAPL & Physical data & SHCA & 1962 & \\
\hline KAPL & Reactor-physics studies with plastic moderator and mock-up & PMA & 1954 & 1966 \\
\hline KAPL & Submarine advanced reactor mock-up & ATR & 1954 & \\
\hline KAPL & SAR physics and mock-up & FPR & 1956 & \\
\hline KAPL & D1G cold-water mock-up & CWA & 1958 & \\
\hline KAPL & SAR high-temperature high-pressure physics and mock-up & PTR & 1958 & \\
\hline KAPL & Cold Water Reactor test assembly & CWTA & 1960 & \\
\hline Lockheed & RER core configurations & CERF & 1958 & \\
\hline LASL, Kiva II & Critical-configuration safety tests & Comet II & 1953 & \\
\hline LASL, Kıva II & Plated bare-plutonium sphere studies & Jezebel & 1954 & \\
\hline LASL, KIva II & $\begin{array}{l}\text { Jacketed enriched-core lateral and base reflector of water } \\
\text { studies, used as neutron sources for exponential column }\end{array}$ & Hydro & 1956 & \\
\hline LASL, Kıva II & Spherical metal cores in thick metal reflector & Flattop & 1957 & \\
\hline LASL, Kıva II & Water-1mmersion safety tests & Water tank & 1961 & \\
\hline Livermore & Critical measurements with different geometries & & 1953 & \\
\hline Livermore & Project PLUTO cold critical experiments & & 1957 & 1964 \\
\hline Livermore & Cold critical experiments for Tory-IIC & & 1963 & 1964 \\
\hline RFP & Critical-configuration safety tests & NSF & 1965 & \\
\hline
\end{tabular}




\section{FOOTNOTES}

1 Power-capacity figures are based on the best avaliable information. In all instances ther mal capacity of the nuclear reactor is given, the electrical output, when shown, is the net is rounded to the nearest hundred low 1s rounded to the nearest hundred is given until the stretch value is approved

2. Thus project is under the Power Demonstration Program

3. The Hallam Nuclear Power Facility was shut down in September 1964 due to moderator can fallures In August 1965 the Commission termmated its contract with Consumer Public Power District for operation of the nuclear plant In May 1966 CPPD turned down their option to purchase the plant In June 1966 the AEC announced deactivation and dis -

mantling of the nuclear facility
4 The Shippingport station is provided with a turbogenerator rated at $90,000 \mathrm{kw}(\mathrm{e})$ net Use of a heat dissipation system permits operation at 150,000 kw(e) gross equivalent on cor 2 Power operation with core 2 began Apr 30,1965

5 The $58,200-\mathrm{kw}(t)$ capacity of the Elk River Reactor 1 in increased to $73,000 \mathrm{kw}(\mathrm{t})$ by a foruivalent to about $16,000 \mathrm{kw}(\mathrm{e})$, the $14,800 \mathrm{kw}(\mathrm{t})$ from the superheater is equivalent about $6000 \mathrm{kw}(\mathrm{e})$

6 In the Consolidated

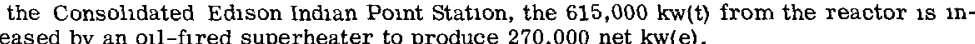

7 In the CVTR plant about $8600 \mathrm{kw}(\mathrm{t})$ is lost to the moderator, and about $300 \mathrm{kw}(\mathrm{t})$ is lost through the piping, this gives a net of $56,000 \mathrm{kw}(\mathrm{t})$ The thermal energy from the reactor is increased to about $66000 \mathrm{kw}(t)$ by an onl-fired superheater to produce 17,000 net $\mathrm{kw}(\mathrm{e})$ Thermal capacity of the reactor is equivalent to about $14,500 \mathrm{kw}(\mathrm{e})$, the $10,000 \mathrm{kw}(\mathrm{t})$ of the superheater is equivalent to about $2500 \mathrm{kw}(\mathrm{e})$.

8 The EGCR project was terminated in January 1966 prior to the completion of construction SRE operated at $20 \mathrm{Mw}(\mathrm{t})$ unt1l shut down in February 1964 for modification to permit an SRE because of limited resources of manpower and funds

10 The bewh was converted to a 100,000 two $(t)$ plant but 1 ts

(1) limited by EBWR in the Bolling Water Program was closed out in December 1962 The reactor is now being used in support of the Plutonium Recycle Program and attained criticality using plutonium as its principal fuel on Sept. 22, 1965 The facility is now planned for use at $40,000 \mathrm{kw}(\mathrm{t})$

11 Current operation of EBR-2 is limited to $45 \mathrm{Mw}(\mathrm{t})$ and 11 to $12 \mathrm{Mw}(\mathrm{e})$ net.

2 In addition to electricity, SURFSIDE is designed to produce 1,000,000 gallons per day of fresh water and 200,000 curies annually of radioactive isotopes

13 This facility was originally bullt and operated in 1954 as the Boiling Reactor Experiment No 2 (BORAX-2) With the addition of a turbogenerator, it operated durng 1955 a BORAX-3, and on July 17, 1955, produced sufficient electricity to light and power Arco, until June 1958, when the experiment was shut down

terphenyls as coolant and/or moderator

15 In a trial run on Dec 21 and 22 1951, EBR-1 generated the world's first electric power from nuclear energy and was the first to demonstrate, in 1953, the feasibility of breeding and the compatibility with breeding economy of sodium-potassium alloy as a liquid-metal coolant. It operated with a plutomum-bearing core (Mark IV) from November 1962 to The reactor was decommissioned and dismantled early in 1964

16 . of construction

18 Typical space propulsion rockets operate for 20 to 40 minutes Therefore space propulsion reactor experiments in context with other reactor applications operate for minutes rather than years.

19 S10FS4 operated in orbit during Apr11-May 1965. Operation terminated unexpectedly after 43 days at power probably due to a sequence of fallures of electrical components of the spacecraft with resulting spurious commands shutting down the reactor An identical ground test unit, s10FS3, operated successfully for more than a year before being shut In 131960 he demonstrated the ability of plutonium fuel elements to perform satısfactorily in a highflux power reactor

21. The SNAPTRAN series of experiments was designed to develop, in a land-based environment, safety information on space auxiliary power reactors through excursion testing at
various temperatures and rates of reactivity insertion. The destructive experiments approach the maximum credible accidents postulated for SNAP reactor systems.

22 A highly enriched plate core was driven to destruction in SPERT-I in the fall of 1962. A low-enriched oxide core successfully resisted destruction in safety tests

24. In 1943 the Manhattan Engineer District disassembled Chicago Pile 1 and rebuilt it at

Palos Park, Ill, as Chicago Ple 2 CP-2 had a thermal-power level of $10 \mathrm{kw}$

25 This reactor was shipped abroad for exhibition purposes in the USAEC Atoms for Peace Exhibit in the Tokyo International Trade Fair in 1959, and in Cairo, Egypt, and Lahore, Pakistan, in 1960

26. This TRIGA-Mk II was operated at the New Delhı Worid Agricultural Fair in 1960 It has

been dismantled for storage in California by GDC
27 In 1965 and 1966 this 1 eactor was operated at Sandia, N Mex , as SNARE Prior to that Reactor (Susie) in the Aircraft Nuclear Propuls Station, Idaho as the Shield Test Pool shut down in 1966 and has been transferred to Loulsiana State University
The BSR-2 which became operable in 1959 is a stanless-steel-UO used alternately in the same facility with BSR-1 (aluminum alloy core)

29 The LITR began as a mechanical model of the MTR After MTR engineering aspects were proved, nuclear fuel, control devices, shielding, and instruments were added, the reactor, then called MTR critical experiment, went eritical for the first time in the spring of 1950 Research facilities were added, and power was increased to the present level in the fall of 1951

30. The AE-6, also designated WBNS, was bult and first operated at Downey Calıf It was moved to Santa Susana in 1956

31 The RER was previously used in the terminated Aircraft Nuclear Propulsion Program A 1icense authorizing Lockheed to operate the reactor as a commercial facility was issued
in July 1962, and in August 1962 the USAF transferred the facility to the General Services Administration. Lockheed acquired title to the facility in March 1965

(Footnotes contanue on the next page) 
32. This TRIGA reactor is capable of being pulsed to peak power levels of approximately $1,200,000 \mathrm{kw}(\mathrm{t})$ for fractions of a second at repeated intervals

33. After the assembly and operation of this reactor in the Government exhibit at Geneva in September 1958, it was dismantled and returned to ANL, where it was rebult as a 250 kw(t) Juggernaut

34 The HPRR was previously operated in the Nevada BREN facllity It is now installed in the Dosimetry Applications Research Facility

35 This reactor was operated in the USAEC Atoms for Peace Exhibit in Viennd, Austria, in June 1963, in Belgrade, Yugoslavia, in September 1963, in Madrid, Spain, in April 1964 in Lisbon, Portugal, in April 1965, in Utrecht, Netherlands, in March 1966, and is scheduled for Dublin Ireland in September-October 1966 and Stockholm, Sweden, in May 1967

36 Th1s reactor was previously designated STF for SNAP Shield Test Facility,

7 AGN-201-112 was operated at the Unity of California, Berkeley, beginning in 1957 The University of New Mexico filed an applic ation in April 1966 for transfer and reconstruction of the reactor at a site on its campus The reactor achieved criticality at the

ACN-201M-105 was previously owned and

8 AGN-201M-105 was previously owned and operated by the National Naval Medical Center sity early in 1964

39 This reactor was originally operated by North Carolina State University as the Raleigh Research Reactor (RRR) It was transferred in March 1966 to Mississippi State University for reactivation The original $10-\mathrm{kw}$ research reactor was started up in 1953 and was dismantled in 1955 The reactor was reactivated with a 500-watt core in March 1957 Early in 1959 the reactor was modified for 100-watt operation and moved to a new location in the Nuclear Scrence Laboratory, and a new type reactor was bult in the original RRR shield vacated by the homogeneous core The RRR was dismantled by N C State in 1963

40 Reactors in the Army Power Program are 1dentified by symbolic nomenclature to reflect mcbility characteristics, power range, development sequence, and field sequence The

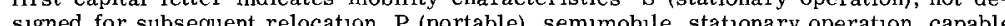
of being dismantled and reassembled for use in successive locations, and $M$ imobilei, capable of being moved intact, or virtually intact, for use in successive locations The second capital letter undicates the power range as measured by design capacity for continuous operation L (low), 100 to $1000 \mathrm{kw}(\mathrm{e}), \mathrm{M}$ (medium) 1000 to $10,000 \mathrm{kw}(\mathrm{e})$, and $\mathrm{H}$ (high), $10,000 \mathrm{kw}(\mathrm{e})$ or more Arabic numerals indicate order in which plants having the same mobility and power characteristics are intiated If not followed by an additional letter, the desiguatron undicates a prototype or pilot plant The last capital letter (when present) indicates the alphabetical order in which field plants of a specific type are initi-

41 The PM-2A was shut down on July 9, 1963, and dismantled during April - June 1964 The reactor vessel was then used by the AEC at the National Reactor Testing Station, Idaho,
for NDT (nil ductility transition temperature) investigations of subected to lone-term ir radition. Defects were seguentially introduced into the vessel wall during a series of tests involving pressure and temperature conditions which exceeded the range permitted in operating nuclear power plants The final test on Nov 18 1966, resulted in a brittle fracture under conditions even more severe that those which had been previously predicted to cause fallure The test program confirmed laboratory data on the adequacy of reactor-operating limitations to prevent brittle fracture of a pressure vessel The remaining parts of the primary system and the secondary system are in storage pending commitment by the Army for final disposition

42 The PM- 1 produces $7 \mathrm{mullion} \mathrm{Btu} / \mathrm{hr}$ for space heating $\mathrm{in}$ addition to electrical output

43. The SM-1A produces $38 \mathrm{milhon} \mathrm{Btu} / \mathrm{hr}$ for space heating in addition to electrical output

44 The MH-1A was installed in the STURGIS (formerly the Liberty Ship CHARLES H CUGLE), at Mobile Ala It has been towed to $\mathrm{Ft}$ Belvoir After acceptance testing in 1966 at Fort Belvoir, Va, the plant will be located at sites as required by the Depart-

45 The USS SEAWOLF, originally commissioned with a sodium-cooled reactor in March 1957, was recommissioned with a pressurized-water reactor on Sept 30, 196

47 In FY 1967 Congress authorized the DLGN originally authorized in FY 1966 The FY 1967 authorization also included long-lead-time procurements for a second, new DLGN
48 The GCRE faclity was first bult and operated to test a gas-cooled light-water-moderated $2200-\mathrm{kw}(\mathrm{t})$ reactor as part of the program to develop the $\mathrm{ML}-1$ Operation of the reactor provide a "dry" facility for testing ML-1 type reactor skids were completed in April 1965.

49 ML-1 operation was terminated and the plant dismantled following a decision in October 1965 to phase out the ML-1 program during FY 1966 Department of the Army is continuing related power-converst

50 The Tory II-C was successfully tested at full design power during May 1964 Subsequent to cancellation of the Pluto program on July 1,1964, the reactor was placed in the Pluto disassembly bullding at NTS for storage

51 Ground-breaking ceremonies for construction of power-conversion equipment by the Washngton Public Power Supply system were held Sept 26, 1963 Initial electric-power

was achieved on Nov 29, 1966 .

The PDP the Reactor facility

In addition to the export power reactors listed, Westinghouse provided the design and for the $115-M w(e)$ Belgium BR-3 pressurnzed-water reactor at Mol

54 This L-77 reactor was operated in the commercial exhibit of the 1958 International ConOctober 1961, in Athens, Greece, in May 1962, and in Bangkok, Thalland, in November 1962. It is presently in storage without fuel at interatom, inc

55 This is the 1955 Geneva Conference reactor rebullt with increased power and now operating at Wuerenlingen, Switzerland

56 The Venezuela Research Reactor was shut down in September 1961 It is expected that the reactor will not be operated again unt1l 1966

This TRIGA reactor was operated at the 1958 International Conference in Geneva prior to Then to the Univerating at the University of Lovanium in June 1959 It is the first reactor to be operated on the African continent

58 The Netherlands research reactor was origually operated at the Amsterdam International Exhibition in June 1957, major portions of the exhibition reactor system were used to fabricate the present reactor

59 This reactor was operated in the International Science Section of the Brussels International Exhibition, Apr 15 to Oct 1, 1958, prior to transfer to the University of Basel

60 The AGN-201-111 was operated first in the USAEC Atoms for Peace Exhibit in Rome, Jtaly, in July 1958 and later in the commercial exhibit of the 1958 International Conference in Geneva prior to transfer to the University of Geneva

61 Prior to its sale to the University of Montevideo in 1966, this reactor was part of the USAEC Exhibit Program It was in Buenos Alres, Argentina, in the fall of 1960 , in Rio de Ju the spraz of 1062 in Sant spring of 1963 , and in Montevideo, Uruguay in the fall of 1963

62 Zero-power experiments of historical interest previously conducted in ANL facility cells include the NAUTILUS core design (ZPR-1), the Savannah River reactor design (ZPR-2), (ZPR-5) The following experiments have been performed in the ZPR- 7 facility thorium, uranium, deuterium criticals (THUD), and a series of flux-trap criticals for the Argonne High Flux Research Reactor

63 The B\&W cells 2 and 3 share a control panel, only one cell can be operated at any one time

64 The NASA ZPR-I and ZPR-II systems will be operated from the same control panel, but it

will be possible to operate only one systern at a time

me operated in elther pot. but not simultaneously 
Single copies of this publication may be obtained free of charge upon request to

\author{
Division of Technical Information Extension \\ U. S. Atomic Energy Commission \\ P. O. Box 62 \\ Oak Ridge, Tennessee 37830
}

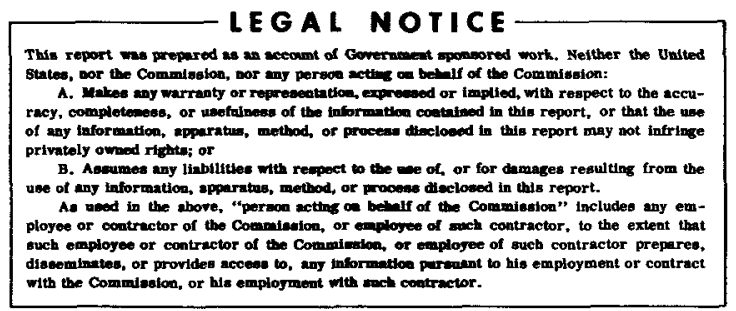

